\title{
Emerging Dissipative Phases in a Superradiant Quantum Gas with Tunable Decay
}

\author{
Francesco Ferri $\odot,{ }^{1}$ Rodrigo Rosa-Medina $\odot,{ }^{1}$ Fabian Finger $\odot,{ }^{1}$ Nishant Dogra $\odot,{ }^{1, *}$ Matteo Soriente $\odot,{ }^{2}$ \\ Oded Zilberberg $\oplus^{2}$ Tobias Donner $\oplus^{1, \dagger}$ and Tilman Esslinger $\oplus^{1}$ \\ ${ }^{1}$ Institute for Quantum Electronics, ETH Zürich, 8093 Zürich, Switzerland \\ ${ }^{2}$ Institute for Theoretical Physics, ETH Zürich, 8093 Zürich, Switzerland
}

(Received 8 April 2021; accepted 1 October 2021; published 7 December 2021)

\begin{abstract}
Exposing a many-body system to external drives and losses can transform the nature of its phases and opens perspectives for engineering new properties of matter. How such characteristics are related to the underlying microscopic processes of the driven and dissipative system is a fundamental question. Here, we address this point in a quantum gas that is strongly coupled to a lossy optical cavity mode using two independent Raman drives, which act on the spin and motional degrees of freedom of the atoms. This setting allows us to control the competition between coherent dynamics and dissipation by adjusting the imbalance between the drives. For strong enough coupling, the transition to a superradiant phase occurs, as is the case for a closed system. Yet, by imbalancing the drives, we can enter a dissipation-stabilized normal phase and a region of multistability. Measuring the properties of excitations on top of the out-ofequilibrium phases reveals the microscopic elementary processes in the open system. Our findings provide prospects for studying squeezing in non-Hermitian systems, quantum jumps in superradiance, and dynamical spin-orbit coupling in a dissipative setting.

DOI: $10.1103 /$ PhysRevX.11.041046

Subject Areas: Condensed Matter Physics,

Quantum Physics
\end{abstract}

\section{INTRODUCTION}

Open many-body systems can annul fundamental laws that typically govern the physics of systems in thermal equilibrium. In the idealized case of an ensemble of interacting particles, isolated from the environment and at zero temperature, the ground state is set by energy minimization, and phase transitions arise from competing energy contributions $[1,2]$. In open systems, however, the interplay between coherent dynamics within the system and its interaction with the environment gives rise to a much richer set of phenomena [3-8]. Such interaction not only is unavoidable, but can be exploited via the engineering of external drives and coupling to specific baths [9-12]. The experimental access to many-body ground states provided by ultracold atoms [13-15] laid the foundation for a recent revival of interest in many-body systems interacting with their environment. Experimental observations that are specifically due to the system's openness

\footnotetext{
*Present address: Cavendish Laboratory, University of Cambridge, J.J. Thomson Avenue, Cambridge CB3 OHE, United Kingdom

†donner@phys.ethz.ch

Published by the American Physical Society under the terms of the Creative Commons Attribution 4.0 International license. Further distribution of this work must maintain attribution to the author(s) and the published article's title, journal citation, and DOI.
}

include the emergence of bistability [16-19], the stabilization of insulating phases [20,21], the access to absorbing-state phase transitions [22], the appearance of dissipation-induced instabilities [23] and time crystals [24], and the change in correlation properties $[25,26]$ that can signal non-Hermitian phase transitions [27].

Besides their fundamental interest, nonequilibrium phenomena bear the prospect of becoming a powerful tool for engineering new materials ranging from exciton condensates to light-induced superconductors [28-32]. The properties of these phases of matter emerge from tuning the elementary excitations by hybridization with the light field $[33,34]$, which provides a natural coupling to the external environment in the presence of optical drives and losses $[35,36]$. To gain further insight into this phenomenology, it is desirable to achieve good experimental control over coherent and dissipative channels and at the same time to gain access to the microscopic properties lying at the origin of the macroscopic phases [37].

In this work, we engineer a driven-dissipative manybody system with global-range interactions that is subject to tunable coherent and dissipative channels. Our realization employs a quantum gas strongly coupled to an optical cavity $[38,39]$. Building on schemes that have been extensively exploited with thermal atoms where the atomic spin is coupled to light fields [40-45], our implementation also involves the density degree of freedom of the gas [46-48]. We employ two Raman laser drives to control the 
strength of the co- and counterrotating terms of the resulting light-matter coupling independently. In combination with photon loss from the cavity, this allows us to explore different regimes of competing coherent coupling and dissipation. Schematically, one can identify the following processes (Fig. 1): The combined action of the two drives coherently mixes two many-body atomic states $\left(\left|0_{a}\right\rangle\right.$

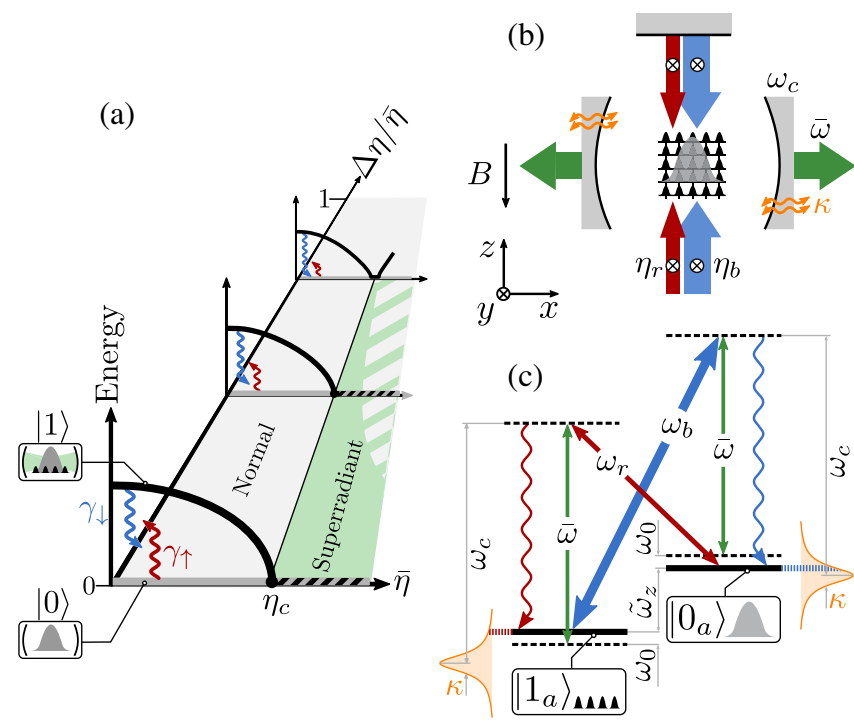

FIG. 1. Competing coherent coupling and dissipation at a superradiant phase transition. (a) A quantum gas interacts coherently with a cavity mode via two tunable drives with mean coupling strength $\bar{\eta}$ and imbalance $\Delta \eta$, giving rise to two lowenergy polariton modes $|0\rangle$ and $|1\rangle$, corresponding to decoupled and coupled light-matter modes, respectively. Increasing $\bar{\eta}$ softens the energy of $|1\rangle$ (black line); cavity dissipation is responsible for the effective damping $\left(\gamma_{\downarrow}\right)$ and amplification $\left(\gamma_{\uparrow}\right)$ of the softmode polariton $|1\rangle$. For small enough $\Delta \eta / \bar{\eta}$, the rates $\gamma_{\downarrow}$ and $\gamma_{\uparrow}$ are balanced, and the mode softening to zero energy at $\bar{\eta}=\eta_{c}$ is accompanied by a phase transition from the normal phase populating only $|0\rangle$ (gray shade) to the superradiant phase (green shade), where $|1\rangle$ is occupied. By increasing $\Delta \eta / \bar{\eta}$, the dominating damping $\gamma_{\downarrow}$ of the soft mode leads first to bistability (green-gray hashed region) and finally to the suppression of the superradiant transition. (b) Sketch of the experimental setup and (c) corresponding level scheme. A BEC inside a high-finesse cavity (with resonant frequency $\omega_{c}$ and field decay rate $\kappa$ ) is illuminated transversally by two Raman lasers with coupling strengths $\eta_{b(r)}$ and frequencies $\omega_{b(r)}$. The BEC $\left(\left|0_{a}\right\rangle\right)$ couples to a spatially modulated state $\left|1_{a}\right\rangle$ of the neighboring Zeeman sublevel, separated by an energy $\hbar \tilde{\omega}_{z}=\hbar\left(\omega_{z}-2 \omega_{\text {rec }}\right)$, with Zeeman splitting $\hbar \omega_{z}$ and recoil energy $\hbar \omega_{\text {rec }}$. In the superradiant phase, a coherent field at frequency $\bar{\omega}$ (green) builds up in the cavity. The two-photon transitions driven by each pump and the cavity field at $\bar{\omega}$ are detuned from the bare atomic states $\left|0_{a}\right\rangle$ and $\left|1_{a}\right\rangle$ by $\mp \omega_{0}$, as indicated by the lower dashed lines. The dissipative channels between modes $|0\rangle$ and $|1\rangle$ shown in (a) are due to Raman scattering of photons from each single drive into the cavity (blue and red wiggly arrows) and subsequent photon loss. The Lorentzian density of states of the cavity is sketched in orange. and $\left.\left|1_{a}\right\rangle\right)$ with the cavity field into polariton modes. As the strength of the drives increases, the excited polariton mode $|1\rangle$ softens to the lowest-energy one $|0\rangle$, and a second-order phase transition occurs from a normal to a superradiant phase that is phase locked to the drives. In addition to this coherent process, each individual drive can induce transitions from one atomic state to the other $\left(\left|0_{a}\right\rangle \leftrightarrows\left|1_{a}\right\rangle\right)$, where photons are scattered into the cavity mode and successively lost. As a result, adjusting the relative strength of the two lasers yields to tuning the effective polariton dissipation. This leads to qualitative changes in the phase diagram of the system, with the appearance of a dissipation-stabilized phase and a discontinuous phase transition in a multistable region.

In our system, the presence of external drives and dissipation leads to complex effects that cannot be interpreted as a simple competition between gain and loss rates, as one would do, for instance, in studying the transition to radiance in a laser. From the point of view of the atomic system, the cavity decay constitutes a nonlocal dissipation channel; this is a crucial feature to obtain the results presented in this work, as detailed in the Appendix D. To explain our observations, we provide a microscopic description of our system and find an effective model that allows us to connect the phase diagram to the underlying properties of the polaritonic excitations, which we characterize experimentally and theoretically.

\section{SETUP AND TUNABLE DECAY}

In our experiments, we trap a Bose-Einstein condensate (BEC) inside a high-finesse optical cavity and pump the atoms with a two-frequency optical standing wave, perpendicular to the cavity axis [Fig. 1(b)]. The BEC is formed by $N=10^{5}$ atoms of ${ }^{87} \mathrm{Rb}$, prepared in the $m_{F}=-1$ sublevel of the $F=1$ ground-state hyperfine manifold. A magnetic field along the $z$ direction is applied to generate a Zeeman splitting $\omega_{z}=2 \pi \cdot 48 \mathrm{MHz}$ between the initial state and the $m_{F}=0$ sublevel. The driving fields are far red detuned from the atomic resonance with frequencies $\omega_{b}$ and $\omega_{r}$ that lie on opposite sides of the dispersively shifted cavity resonance $\omega_{c}$, i.e., $\omega_{r}<\omega_{c}<\omega_{b}$, and $\omega_{b}-\omega_{r} \sim 2 \omega_{z}$. The standing-wave modulations of the two drives overlap at the position of the atoms, forming a one-dimensional lattice potential with spacing $\lambda / 2=784.7 / 2 \mathrm{~nm}$. Each pump beam realizes a cavity-assisted Raman coupling between the $m_{F}=-1$ and $m_{F}=0$ levels, as sketched in Fig. 1(c). The resulting system is effectively described using two atomic modes possessing both a well-defined spin and motional state: the initial ground state of the trapped BEC $\left|0_{a}\right\rangle$, and the excited-momentum state of the neighboring Zeeman sublevel $\left|1_{a}\right\rangle \propto \cos \left(k_{\text {rec }} \hat{x}\right) \cos \left(k_{\text {rec }} \hat{z}\right) \hat{F}_{+}\left|0_{a}\right\rangle$, with $\hat{F}_{+}$being the raising spin operator in the $F=1$ manifold and $\hbar k_{\text {rec }}=2 \pi \hbar / \lambda$ the recoil momentum. When increasing the driving strength, the ground state $\left|0_{a}\right\rangle$ evolves from a 
harmonically confined BEC to a stack of pancake-shaped BECs trapped in the maxima of the standing-wave drives.

In a rotating frame defined by the driving frequencies, the coherent dynamics of our system is described by the many-body Hamiltonian

$$
\begin{aligned}
\hat{H}= & -\hbar \Delta_{c} \hat{a}^{\dagger} \hat{a}+\hbar \omega_{0} \hat{J}_{z}+2 \hbar \bar{\eta}\left(\hat{a}+\hat{a}^{\dagger}\right) \hat{J}_{x} \\
& +2 i \hbar \Delta \eta\left(\hat{a}-\hat{a}^{\dagger}\right) \hat{J}_{y},
\end{aligned}
$$

as detailed in Appendix B. Here, $\hat{J}_{i=x, y, z}$ are the components of the pseudospin operator associated with the two many-body states $\left|0_{a}\right\rangle \quad\left(\left\langle\hat{J}_{z}\right\rangle=-N / 2\right)$ and $\left|1_{a}\right\rangle$ $\left(\left\langle\hat{J}_{z}\right\rangle=N / 2\right)$, and $\hat{a}\left(\hat{a}^{\dagger}\right)$ is the annihilation (creation) operator of the relevant cavity mode. The effective atomic frequency is $\omega_{0}=\left(\omega_{b}-\omega_{r}\right) / 2-\omega_{z}+2 \omega_{\text {rec }}$, with the recoil energy $\hbar \omega_{\text {rec }}$ and the cavity detuning $\Delta_{c}=\bar{\omega}-\omega_{c}$, where $\bar{\omega}=\left(\omega_{b}+\omega_{r}\right) / 2$ is the mean of the pump frequencies. The light-matter coupling strengths are parametrized by $\bar{\eta}=\left(\eta_{b}+\eta_{r}\right) / 2$ and $\Delta \eta=\left(\eta_{b}-\eta_{r}\right) / 2$, with the cavityassisted Raman coupling $\eta_{b(r)}$ arising from the $\omega_{b(r)}$ pump and the cavity mode. These two-photon Raman couplings implement the independently tunable co- and counterrotating terms of the light-matter interaction. The dynamics of the open system due to photon losses is well described by a master equation $\dot{\hat{\rho}}=-i / \hbar[\hat{H}, \hat{\rho}]+$ $\hat{\mathcal{L}}[\hat{\rho}]$, where the Lindblad superoperator $\hat{\mathcal{L}}[\hat{\rho}]=\kappa\left[2 \hat{a} \hat{\rho} \hat{a}^{\dagger}-\right.$ $\left.\left\{\hat{a}^{\dagger} \hat{a}, \hat{\rho}\right\}\right]$ accounts for the cavity field decay at rate $\kappa=2 \pi \cdot 1.25 \mathrm{MHz}$. The model introduced here is a generalized Dicke model that is predicted to exhibit rich phenomenology [8,49-52]. Correspondingly, first experiments exploring effective Dicke models with tunable coand counterrotating terms and different beam geometries have been carried out using thermal atoms [42,53], whose motional state is not well defined.

When the co- and counterrotating coupling terms are balanced $(\Delta \eta=0)$, Eq. (1) reduces to the Dicke Hamiltonian [54,55]. In this limit, at low coupling $\bar{\eta}$, the system is in the normal phase with $\left\langle\hat{J}_{x}\right\rangle=0,\langle\hat{a}\rangle=0$, and the lowest-energy polariton mode $|0\rangle$ is mostly occupied. By increasing the coupling $\bar{\eta}$, the energy of the first excited polariton $|1\rangle$, admixing both atomic states $\left|0_{a}\right\rangle$ and $\left|1_{a}\right\rangle$ and the cavity photons, softens. As the energy of the polariton |1) reaches zero, the system undergoes a second-order phase transition to the self-organized superradiant phase with $\left\langle\hat{J}_{x}\right\rangle \neq 0,\langle\hat{a}\rangle \neq 0$ [Fig. 1(a)] [39,56]. In real space, the superradiant phase exhibits a density modulation with period $\lambda / 2$ along the $x$ and $z$ direction, while the $x$ component of the spin is staggered in a checkerboard modulation with period $\lambda$ [46]. The transition occurs at a collectively enhanced critical coupling $\eta_{c} \sqrt{N}=$ $\sqrt{-\omega_{0}\left(\Delta_{c}^{2}+\kappa^{2}\right) /\left(4 \Delta_{c}\right)}$, which is only slightly shifted from the closed-system critical point for our parameters; see Appendix C. On the other hand, if an imbalance $\Delta \eta$ between the coupling of co- and counterrotating terms is introduced, the effect of dissipation on the system becomes qualitatively different. Specifically, due to cavity loss, each Raman drive $\eta_{b(r)}$ is responsible for an effective decay $\gamma_{\downarrow(\uparrow)}$ of the polariton mode $|1\rangle(|0\rangle)$ toward mode $|0\rangle(|1\rangle)$. In the parameter regime of our experiment $\omega_{0} \ll \kappa$, the effective decay rates take the form

$$
\gamma_{\downarrow(\uparrow)}=N \frac{\kappa}{\Delta_{c}^{2}+\kappa^{2}} \eta_{b(r)}^{2},
$$

which we derive in Appendix D using an effective Keldysh action for the polariton modes. We identify that the microscopic process corresponding to the effective decay $\gamma_{\downarrow(\uparrow)}$ is a collectively enhanced Raman scattering driven by the $\omega_{b(r)}$ pump beam, into the dissipatively broadened density of states of the cavity, as sketched in Fig. 1(c). This mechanism is analogous to the Raman decay lying at the heart of superradiant Raman lasers [57-60]. Note that the effective decays (2) are independent of the phase of the cavity field. This is different from the coherent Raman couplings leading to the superradiant phase, where the intracavity field is always in or out of phase with the effective driving field $\bar{\omega}$ [61]. The two processes $\gamma_{\downarrow(\uparrow)}$ are mediated by the same cavity mode, giving rise to effective nonlocal decay and amplification channels for the population of mode $|1\rangle$. This allows the two processes to compensate each other when the Raman drives are balanced in strength or to globally enhance the dissipative effects for imbalanced drives (see Appendix D). In particular, as we experimentally demonstrate, the effective damping generated by these processes leads to a dramatic modification of the superradiant phase transition, as well as to new regions of multistability and hysteresis.

\section{MEASUREMENT OF THE PHASE DIAGRAM}

We restrict the experiments to the parameter space $0 \leq \Delta \eta \leq \bar{\eta}$; the properties of the system in the region $0 \leq \bar{\eta} \leq \Delta \eta$ are mirrored-cf. Eq. (1). We map out the phase diagram of the system by ramping up the power of the pump beams while keeping the ratio $\Delta \eta / \bar{\eta}$ constant and monitoring the cavity output with a heterodyne detection; see Fig. 2(a). The onset of a superradiant phase is signalled by the buildup of a coherent cavity field with frequency $\bar{\omega}$ above a critical coupling strength. We show in Fig. 2(b) the measured mean intracavity photon number $n_{\mathrm{ph}}$ in the $(\bar{\eta}, \Delta \eta)$ parameter space. At small imbalances $\Delta \eta \ll \bar{\eta}$, the phenomenology of the Dicke phase transition is observed, and the value of the coupling $\bar{\eta} \approx \eta_{c}$ at which the transition occurs depends only weakly on $\Delta \eta$. In contrast, at larger ratios $\Delta \eta / \bar{\eta}>0.71(2)$, the superradiant phase transition is suppressed, and the system remains in the normal phase at values of $\bar{\eta}$ largely above $\eta_{c}$. We compare the measured critical couplings with the phase boundaries obtained from a mean-field treatment of our driven-dissipative model and 


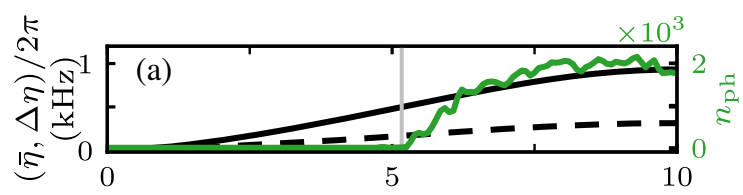

Time (ms)

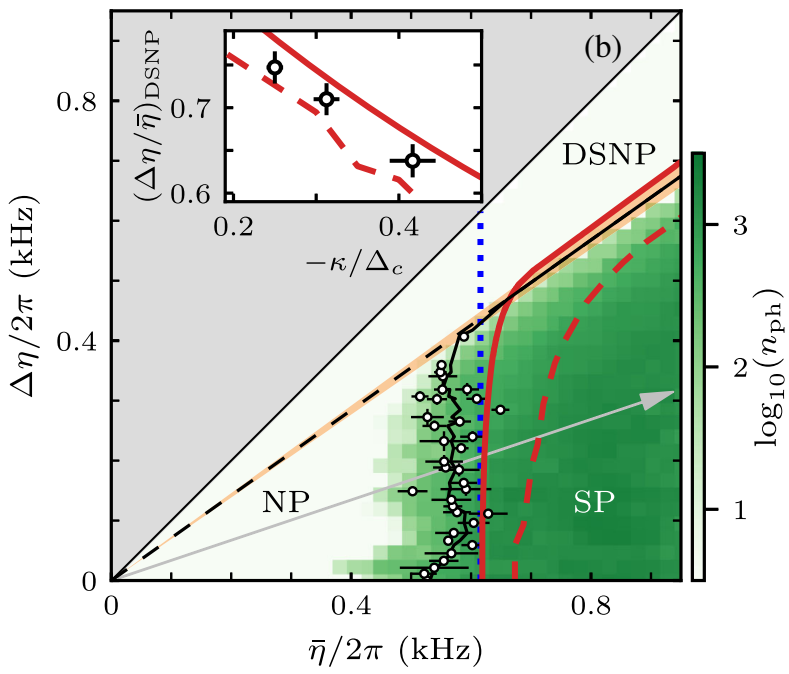

FIG. 2. Phase diagram. (a) Experimental protocol. We ramp up the couplings $\bar{\eta}$ (solid black line) and $\Delta \eta$ (dashed black line) within $10 \mathrm{~ms}$ at constant ratio $\Delta \eta / \bar{\eta}$. A typical time trace of the mean intracavity photon number $n_{\mathrm{ph}}$ for $\Delta \eta / \bar{\eta}=0.34$ is shown (green line). For any $\Delta \eta / \bar{\eta}<0.71(2)$, a coherent cavity field builds up in the cavity (superradiant phase) above a critical coupling (gray vertical line). (b) Green: intracavity photon number $n_{\text {ph }}$ as a function of the couplings $\bar{\eta}$ and $\Delta \eta$, obtained by implementing the protocol shown in (a) for 51 different values of $\Delta \eta / \bar{\eta}$ and averaging over five repetitions. Labels NP, SP, and DSNP stand for normal, superradiant, and dissipationstabilized normal phase, respectively. The dots indicate the critical point extracted from the measured photon traces and averaged within each subset of constant $\Delta \eta / \bar{\eta}$ with the error bars representing the standard error of the mean (SEM). The slope $(\Delta \eta / \bar{\eta})_{\text {DSNP }}$ of the dashed line corresponds to the smallest value of $\Delta \eta / \bar{\eta}$ at which the SP is not present (the uncertainty on the slope is marked as a shaded orange region around the line). The black line is a guide to the eye through the critical points (obtained as a sliding average over seven points) and along the measured boundary of the DSNP. We mark the boundary of the SP obtained from a mean-field stability analysis (solid red line) and from a numerical simulation of the measurement protocol (dashed red line) [8]. For comparison, the analytical mean-field result for the closed system is also plotted (blue dotted line). The arrow indicates the measurement path followed in (a). Inset: slope $(\Delta \eta / \bar{\eta})_{\text {DSNP }}$ extracted from phase diagrams measured at different cavity detunings $\Delta_{c}$ and plotted vs $-\kappa / \Delta_{c}$. The upper (lower) vertical error bar corresponds to the smallest (largest) value for $\bar{\eta} / \Delta \eta$ at which the phase transition is always absent (present), while the horizontal ones arises from the uncertainty of $\Delta_{c}$. The lines are predictions from analytical (solid) and numerical (dashed) calculations. For this measurement, $N=1.28(8) \times 10^{5}, \omega_{0}=2 \pi \cdot 44(2) \mathrm{kHz}$, and, in (a) and (b), $\Delta_{c}=-2 \pi \cdot 4.0(2) \mathrm{MHz}$. observe consistency between the experiment and our theoretical description. The phase boundaries are calculated from both the steady-state solutions and numerical simulations including time-varying couplings, with no free parameters (see Appendix $\mathrm{C}$ for details).

The existence of a dissipation-stabilized normal phase near the line $\Delta \eta / \bar{\eta}=1$ is a consequence of the open character of our system, as pointed out in previous theoretical works $[8,50]$. Specifically, the superradiant phase originates from the coupling between the cavity and the pseudospin of the atomic ensemble that, above a critical value, pushes the normal phase to become an unstable excited state. As a crucial ingredient of our system, photon decay leads to an effective loss channel that stabilizes the normal phase, giving rise to a population inversion scenario [52]. To further characterize the dissipation-stabilized normal phase, we measure the full phase diagram of the system for different cavity detunings $\Delta_{c}$. We observe that the slope $(\Delta \eta / \bar{\eta})_{\text {DSNP }}$ of the boundary between the dissipation-stabilized normal phase and the superradiant phase decreases for smaller cavity detunings [inset in Fig. 2(b)]. This agrees with the predictions of our theoretical model, by which we find that in the limit of large coupling $\bar{\eta} \gg \eta_{c}$ the slope of the phase boundary is given by $(\Delta \eta / \bar{\eta})_{\mathrm{DSNP}}=\kappa / \Delta_{c}\left(1-\sqrt{1+\Delta_{c}^{2} / \kappa^{2}}\right)$; see Appendix C.

\section{PROBING THE EXCITATION SPECTRUM}

The effective damping induced by the relative coupling imbalance $\Delta \eta / \bar{\eta}$ leads to a profound change in the superradiant phase transition and even suppresses it. This observation is closely linked to the system's spectrum of collective excitations. We implement an experimental protocol that allows for nondestructive, real-time monitoring of the free evolution of the excited polariton mode $|1\rangle$, both in amplitude and in frequency. We promote a small population of mode $|0\rangle$ to mode $|1\rangle$ by means of a Bragg scattering process involving the transverse pump beams and a 1-ms-long laser pulse injected into the cavity at frequency $\bar{\omega}+\omega_{0}+\delta_{\text {probe }}$. This small occupancy of the excited mode produces a scattering of a weak field from the pumps into the cavity also after the pulse ends, which we monitor with the frequency-resolving heterodyne detector [62]. We shine the excitation pulse at a fixed time while ramping up both couplings at constant ratios $\Delta \eta / \bar{\eta}$. At the falling edge of the pulse, the mean pump intensities correspond to $\bar{\eta} / \eta_{c} \approx 0.6$ [Fig. 3(a)].

In Figs. 3(b)-3(e), we present single-shot spectrograms of the cavity field showing the excitation pulse and the subsequent evolution of the system for increasingly larger values of $\Delta \eta / \bar{\eta}$. In the Dicke limit $[\Delta \eta / \bar{\eta}=0$, Fig. 3(b)], the main components of the spectrum evolve toward $\bar{\omega}$ as the coupling $\bar{\eta}$ is swept to larger values, reflecting the softening of the excited mode energy. At the critical point $\bar{\eta}=\eta_{c}$, the energy gap between mode $|0\rangle$ and the soft 

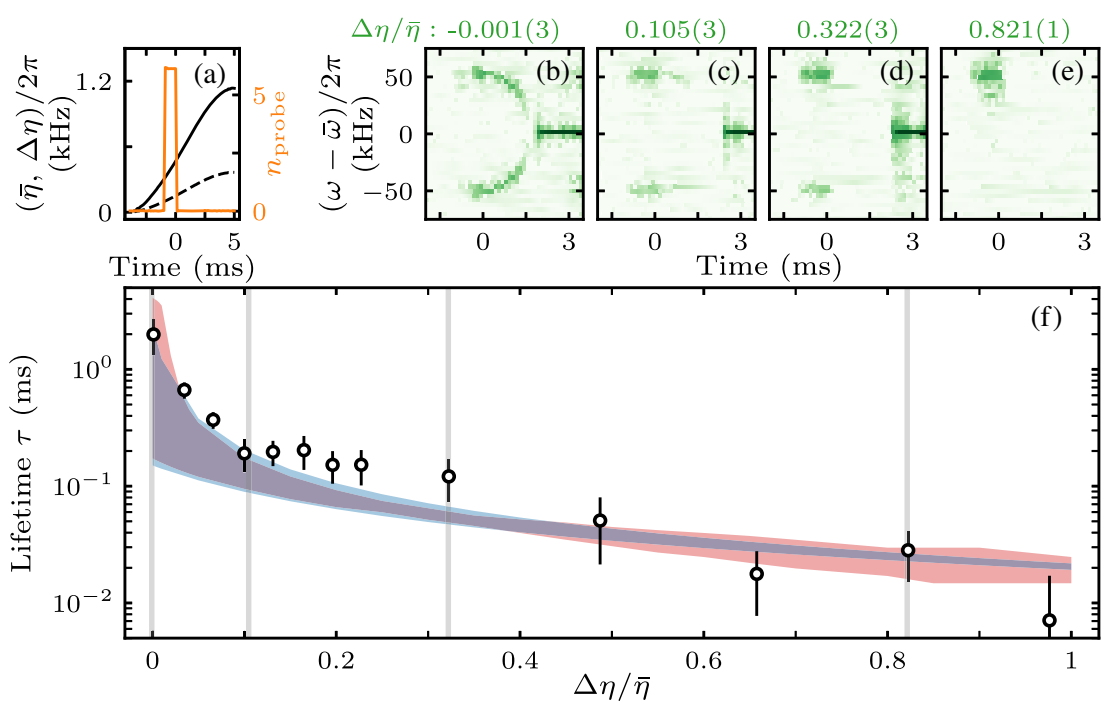

(f)



FIG. 3. Properties of the excited polariton. (a) Experimental protocol. While ramping up the couplings $\bar{\eta}$ (solid black line) and $\Delta \eta$ (dashed line) at constant $\Delta \eta / \bar{\eta}$, we populate the excited mode $|1\rangle$ using a weak excitation pulse along the cavity axis (orange line). Throughout this measurement, the maximal coupling $\bar{\eta}$ at the end of the ramp is $2 \pi \cdot 1.16(5) \mathrm{kHz}$. (b)-(e) Representative heterodyne spectrograms for different ratios $\Delta \eta / \bar{\eta}$, showing the frequency-resolved mean number of photons $\tilde{n}_{\mathrm{ph}}$, as a function of the frequency and time. In (e), the large imbalance $\Delta \eta / \eta$ suppresses the superradiant phase transition. (f) Data points: measured lifetime of the induced excitations as a function of $\Delta \eta / \bar{\eta}$. The error bars showcase the maximum between the SEM and the time resolution of the photon traces $(10 \mu \mathrm{s})$. Blue shaded region: predicted lifetime from the analytical eigenvalues [cf. $(\mathrm{g})$ and $(\mathrm{h})]$, assuming a phenomenological atomic dephasing between 0 (upper bound) and $2 \pi \cdot 500 \mathrm{~Hz}$ (lower bound); the latter is of the order of the $s$-wave scattering rate. Red shaded region: numerical simulation results. The values of $\Delta \eta / \bar{\eta}$ shown in (b)-(e) are marked with gray vertical lines. (g),(h) Excitation eigenvalues of the system, linearized around the normal phase, and assuming zero atomic dephasing. Colors indicate different $\Delta \eta / \bar{\eta}$ values; line shape indicates stable normal phase (solid), stable superradiant phase (dotted), and bistability (dashed). For the results presented here, $N=9.6(4) \times 10^{4}, \omega_{0}=2 \pi \cdot 48(4) \mathrm{kHz}, \Delta_{c}=-2 \pi \cdot 5.8(1) \mathrm{MHz}$, and $\delta_{\text {probe }}=2 \pi \cdot 2.0(4) \mathrm{kHz}$.

mode $|1\rangle$ vanishes, and the superradiant phase transition occurs, signalled by the buildup of a strong coherent field at frequency $\bar{\omega}$. As the relative imbalance $\Delta \eta / \bar{\eta}$ is increased, the mode softening is accompanied by a faster decay of the excitation amplitude during the experiment. The $1 / e$ lifetime $\tau$ of the free excitation extracted from the integrated spectrograms decreases rapidly as the relative imbalance $\Delta \eta / \bar{\eta}$ is increased [Fig. 3(f)]. At the same time, the superradiant phase transition occurs at a coupling $\bar{\eta}$ that depends only weakly on the coupling imbalance $\Delta \eta$ until, for large enough $\Delta \eta / \bar{\eta}$, the transition is fully suppressed.

The connection between the damping of the excitations and the suppression of the superradiant phase transition can be understood by analyzing the excitation spectrum of the open system. We linearize the mean-field equations of motion around the normal phase and study the low-energy eigenfrequencies $\omega_{ \pm}$of the corresponding dynamical matrix, as a function of the coupling $\bar{\eta} / \eta_{c}$ and for different values of $\Delta \eta / \bar{\eta}$ [Figs. 3(g) and 3(h)]. The real part of the spectrum captures the energy of the excited polariton, while a negative (positive) imaginary part signals damping (amplification). At first order in $\omega_{0} / \kappa \ll 1$, the eigenfrequencies are given by

$$
\omega_{ \pm}=-i\left(\gamma_{\downarrow}-\gamma_{\uparrow}\right) \pm \omega_{0} \sqrt{\left(1-\frac{\bar{\eta}^{2}}{\eta_{c}^{2}}\right)\left(1-\frac{\Delta \eta^{2}}{\eta_{c}^{2}}\right)},
$$

as illustrated in Appendix D. As the coupling $\bar{\eta}$ increases toward the critical point $\eta_{c}$, the phase transition occurs whenever any of $\operatorname{Im}\left[\omega_{ \pm}\right]$becomes positive $[55,63,64]$. At large enough ratios $\Delta \eta / \bar{\eta}$, the damping rate $\gamma_{\downarrow}$ of the soft mode is dominant; this counteracts the coherent buildup of superradiance and stabilizes the normal phase.

We report in Fig. 3(f) (blue shaded region) an estimate of the quasiparticle lifetime $\tau=-\left(2 \operatorname{Im}\left[\omega_{ \pm}\right]\right)^{-1}$ obtained from the spectrum of the eigenvalues. For this estimation, we assume that the couplings $\bar{\eta}$ and $\Delta \eta$ are kept fixed at the end of the excitation pulse; this simplification provides a valid approximation for the measured lifetime where $\tau$ varies only slightly during the decay, i.e., as long as $\Delta \eta / \bar{\eta} \gtrsim 0.05$. For a closer comparison with the experimental data, we perform a numerical simulation of our experimental protocol including the excitation pulse [red shade in Fig. 3(f)]. To account for collisional interactions and spin dephasing, the theoretical estimations include a phenomenological atomic damping (see Appendix D), which we assume constant throughout the dynamics. 


\section{BISTABILITY AND HYSTERESIS}

We focus now on the boundary between the superradiant phase and the dissipation-stabilized normal phase observed at large imbalances $\Delta \eta / \bar{\eta}$. Previous theoretical works predict an intermediate region where both phases are stable (bistability) [8,50]. Accordingly, the occurrence of a firstorder phase transition is expected. The bistability can be understood in terms of a competition between the coherent and dissipative processes described above. By increasing the relative imbalance $\Delta \eta / \bar{\eta}$, the damping $\gamma_{\downarrow}$ fosters the population of mode $|0\rangle$ and acts against the coherent coupling responsible for superradiance. In the limit of dominant dissipation, such damping makes the dissipationstabilized normal phase the unique stable steady state. Conversely, in the regime of comparable coherent coupling and dissipation, the phase to which the system converges depends on its initial preparation. If the system is prepared


FIG. 4. Hysteresis at the boundary between the superradiant and the dissipation-stabilized normal phase. (a) Time trace of the intracavity photons in a single typical experimental realization, when crossing the boundary between the superradiant and the dissipation-stabilized normal phase in opposite directions. (b) Corresponding trajectory in the parameter space $(\bar{\eta}, \Delta \eta)$. The hysteresis is measured at constant $\bar{\eta}$; an artificial offset along $\bar{\eta}$ is introduced between the forward and backward path in the conceptual figure (b) for better visibility. The dashed arrow marks the preparation of the system within $6 \mathrm{~ms}$ in the dissipationstabilized normal phase, starting from zero coupling. Each (down, up) sweep of $\Delta \eta$ across the bistability region (purple, orange) is a 3-ms-long S-shaped ramp. (c) Mapping the hysteresis region. Dots: phase boundary detected during the forward (purple) and backward (orange) path for different $\bar{\eta}$. The positions of the boundaries are determined from the photon traces by setting a threshold of 36 mean intracavity photons, as indicated in (a) with the gray line. The data points shown in (c) are mean values of 12-18 realizations, with the error bars representing the standard deviation. As a guide to the reader, in the background of (b) and (c), the analytical phase diagram highlights the region of stable normal phase (white), stable superradiant phase (dark green), and bistability (light green). The theoretical boundaries are rescaled to the experimental data, with a single factor applied to both couplings. For this measurement, $N=1.10(8) \times 10^{5}$, $\omega_{0}=2 \pi \cdot 40(5) \mathrm{kHz}$, and $\Delta_{c}=-2 \pi \cdot 3.0(5) \mathrm{MHz}$. in the normal phase, it remains stable because of the aforementioned damping. On the other hand, if the system is initially in the superradiant phase, the dissipative damping toward mode $|0\rangle$ is counteracted by the presence of a coherent intracavity field that contributes to keep mode $|1\rangle$ significantly populated. In other words, preparing the system in the organized, symmetry-broken superradiant phase makes it more rigid against transitions assisted by cavity dissipation.

To explore the boundary between the dissipationstabilized normal phase and the superradiant phase, we initialize the system in the former phase by preparing the BEC and ramping up the coupling $\bar{\eta}$ above $\eta_{c}$ at fixed imbalance $\Delta \eta / \bar{\eta}=0.78$. From this initial state, the transition to the superradiant phase is crossed by reducing $\Delta \eta$ at constant $\bar{\eta}$. Then, within the same experimental realization, the direction of the $\Delta \eta$ sweep is reversed and the dissipation-stabilized normal phase is retrieved. The comparison between the forward and backward paths shows a hysteretic behavior at the phase boundary, in agreement with the expected discontinuous character of the transition; see Fig. 4. By performing the hysteresis measurement at different couplings $\bar{\eta}$, an experimentally accessible region where the superradiant and the dissipation-stabilized normal phase are both stable is mapped out [Fig. 4(c)]. We verify that implementing the parameter loop in the opposite direction also gives rise to hysteresis.

\section{CONCLUSION AND OUTLOOK}

We show that varying the imbalance of co- and counterrotating coupling terms between a quantum gas and a lossy optical cavity engenders a tunable competition between coherent and dissipative processes across the superradiant phase transition, leading to the emergence of a dissipationstabilized phase and hysteresis. Combining the control over dissipative and coherent couplings with real-time access to the dynamics allows us to identify the underlying microscopic processes determining the observed phase diagram. We note that, if the cavity dynamics is adiabatically traced out, our system maps to a driven-dissipative version of the anisotropic Lipkin-Meshkov-Glick model [65-69]-an important reference model for quantum magnetism that describes a many-body spin system with all-to-all interactions. Furthermore, interesting phenomena are expected near the boundary of the normal phase [52]; as visible in Figs. 3(g) and 3(h), here the real parts of the eigenfrequencies of the low-lying polariton merge, and their imaginary part bifurcates such that one squeezes, while the other broadens. In the Dicke limit, this phenomenon is limited to the coupling interval between the bifurcation and the transition point to the superradiant phase, which is very narrow for typical experimental parameters [55]. Squeezing of fluctuations can be obtained on a much wider range of parameters at the boundary between the normal phase and the dissipation-stabilized normal phase, where dissipation 
suppresses the change of the system's steady state. Characterizing the fluctuations of the normal modes in this regime can shine light on the generation of squeezing in the vicinity of exceptional points in non-Hermitian systems [70-72]. Moreover, performing experiments with small atom or photon numbers would unveil effects beyond mean field, such as quantum jumps in the bistability region, as predicted recently in Refs. [73,74]. Furthermore, combining our findings on prominent dissipative effects with the generation of cavity-mediated spin-orbit interaction $[75,76]$ opens a way to study spinorbit coupling in a dissipative setting.

\section{ACKNOWLEDGMENTS}

We are grateful to M. Landini and K. Kroeger for contributions at early stages of the experiment, to T. L. Heugel, R. Chitra, A. Eichler, and F. Piazza for fruitful discussions, and to A. Frank for electronic support. F. F., R. R.-M., F. F., T. D., and T. E. acknowledge funding from the Swiss National Science Foundation: Projects No. 182650 and No. 175329 (NAQUAS QuantERA) and NCCR QSIT, from EU Horizon2020: ERCadvanced grant TransQ (Project No. 742579). M. S. and O. Z. acknowledge financial support from the Swiss National Science Foundation through Grants No. PP00P2_1163818 and No. PP00P2_190078, as well as the ETH Research Grant No. ETH-4517-1.

F. F. and R. R.-M. contributed equally to this work.

\section{APPENDIX A: EXPERIMENTAL DETAILS}

\section{BEC preparation and Zeeman shift characterization}

We prepare a BEC of ${ }^{87} \mathrm{Rb}$ atoms in the $m_{F}=-1$ Zeeman sublevel in the $F=1$ hyperfine manifold of the $5^{2} S_{1 / 2}$ electronic level, using radio-frequency-assisted evaporation in a magnetic quadrupole trap. The atom cloud is optically transported and confined at the center of the cavity mode by an optical crossed dipole trap $V_{\text {ext }}$, with frequencies $\left[\omega_{h x}, \omega_{h y}, \omega_{h z}\right]=2 \pi \cdot[220(3), 24.6(8)$, 170.1(3)] Hz. More information on the geometry of the BEC and finite size effects is given in Appendix B 3.

We apply a magnetic field $\mathbf{B}=B_{z} \mathbf{e}_{z}$, with $B_{z}<0$. To measure the Zeeman shift $\omega_{z}$ between the sublevels $m_{F}=-1$ (high-energy level) and $m_{F}=0$ (low-energy level), we employ cavity-assisted Raman transitions. We illuminate the BEC with the transverse pump with frequency $\omega_{r}<\omega_{c}$. Close to the two-photon resonance $\omega_{r} \approx \omega_{c}-\omega_{z}$, a large fraction of the BEC is transferred to $m_{F}=0$ via two-photon processes involving absorption of photons from the red pump and emission into the cavity mode, with an increase of the kinetic energy by $2 \hbar \omega_{\text {rec }}$. In this Raman process, light is scattered into the cavity at frequency $\tilde{\omega}=\omega_{r}+\left(\omega_{z}-2 \omega_{\text {rec }}\right)$, fulfilling energy conservation. We infer the Zeeman splitting $\omega_{z}$ by measuring the frequency $\tilde{\omega}$ of the photons leaking out of the cavity using a heterodyne detection scheme.

\section{Optical cavity}

The optical cavity is a quasi-planar and symmetric Fabry-Perot resonator with a length of $176 \mu \mathrm{m}$ and finesse $\mathcal{F}=3.5 \times 10^{5}$. The $\mathrm{TEM}_{00}$ mode has a waist size $w_{c}=25 \mu \mathrm{m}$. At the frequency of the cavity resonance $\omega_{c}$ employed in our experiment, the amplitude of the electric field per photon is $E_{0}=403 \mathrm{~V} / \mathrm{m}$.

The cavity is actively stabilized by locking the resonance to the frequency of a reference laser with a wavelength of $830 \mathrm{~nm}$, far from atomic resonance, using a Pound-DreverHall technique. The intracavity intensity of the reference laser corresponds to a lattice depth below $0.01 \hbar \omega_{\text {rec }}$, which has negligible influence on the dynamics of the BEC in our experiment. The 830-nm laser is referenced to the laser generating the transverse pump beams via locking on a transfer cavity.

\section{Transverse pump characterization}

The two transverse pumps (drives) are derived from the same laser source. Their frequencies $\omega_{r}$ and $\omega_{b}$ are independently adjusted by employing double-pass acoustic optical modulators (AOMs) in separate optical paths and recombining the beams afterward on the same optical table. The combined fields are then sent to the experiment sharing the same optical fiber to ensure high relative phase stability. A small fraction of the beam is split close to the vacuum chamber and overlapped with an optical local oscillator at frequency $\omega_{\mathrm{LO}}$, also derived from the same laser, on an ac photodiode. The beat notes at frequencies $\omega_{r}-\omega_{\mathrm{LO}}$ and $\omega_{b}-\omega_{\mathrm{LO}}$ are electronically separated and employed for intensity stabilization of the individual pumps. The distance between the retroreflecting mirror and the atomic cloud is carefully adjusted such that the two standing waves overlap maximally at the position of the atomic cloud. The lattice depth associated to each pump is calibrated by means of Kapitza-Dirac diffraction [77]. For all the measurements presented in the main text, we increase the transverse pump powers via S-shaped ramps of the form $V_{r, b}(t)=\tilde{V}_{r, b}\left[3\left(t / t_{\text {ramp }}\right)^{2}-2\left(t / t_{\text {ramp }}\right)^{3}\right]^{2}$, where $\tilde{V}_{r, b}$ is the final lattice depth of the $\omega_{r, b}$ pump and $t_{\text {ramp }}$ is the duration of the ramp.

\section{Heterodyne detection}

We monitor the photon field leaking out of the cavity by separating on a polarizing beam splitter (PBS) the $y$ - and $z$-polarization modes and detecting each of them with heterodyne setups. The detection branch for the $z$ polarization is used to produce the data discussed in this work. The auxiliary detection setup for the $y$-polarized mode is used to probe the cavity resonance at the end of each experimental cycle.

In the heterodyne setup for the relevant $z$-polarized mode, the light field from the cavity is interfered with a local oscillator laser at frequency $\omega_{\mathrm{LO}}$, and the high 
detection bandwidth of $250 \mathrm{MS} / \mathrm{s}$ allows for an all-digital demodulation of the beat note at $\omega-\omega_{\mathrm{LO}}$ over a broad frequency range of $[0,125] \mathrm{MHz}$. In order to calibrate the photon number, we inject an on-resonance laser field into the empty cavity. We find the conversion factor between the demodulated heterodyne signal and the mean intracavity photon number by measuring the power after the PBS with a power meter and using the knowledge on the losses of the cavity mirrors.

The complex intracavity field $\alpha(t)=X(t)-i Y(t)$ is obtained from the quadratures $X(t)$ and $Y(t)$ after digital demodulation at frequency $\delta \omega_{D}=\bar{\omega}-\omega_{\mathrm{LO}}$. The power spectral density (PSD) is calculated as $\operatorname{PSD}(f)$ $=|\operatorname{FFT}(\alpha)|^{2}(f)$ using a fast Fourier transform of the form $\operatorname{FFT}(\alpha)(f)=d t / \sqrt{N} \sum_{i} \alpha^{*}\left(t_{i}\right) e^{-i 2 \pi f t_{i}}$ [23], where $t_{i}$ is the time of the $i$ th step and $N$ is the total number of steps in the integration window. To construct the spectrograms, the traces are divided in time intervals of $T=150 \mu \mathrm{s}$ with an overlap of $50 \%$ between subsequent intervals. Finally, we calculate the photon number spectrograms as $\tilde{n}_{\mathrm{ph}}=\operatorname{PSD}(f) / T$.

\section{APPENDIX B: DERIVATION OF THE HAMILTONIAN}

In this Appendix, we derive the Hamiltonian in Eq. (1) from the closed-system dynamics of our spinor BEC coupled to the cavity.

\section{Single-atom Hamiltonian}

The Hamiltonian of a single atom coupled to the cavity mode reads

$$
\hat{H}_{1}^{\prime}=\hat{H}_{\mathrm{at}}^{\prime}+\hat{H}_{\mathrm{cav}}^{\prime}+\hat{H}_{\mathrm{int}}^{\prime} .
$$

In the dispersive regime of atom-light interaction [78,79], the optically excited atomic states of the atom can be adiabatically eliminated, and the Hamiltonian of the bare atom $\hat{H}_{\text {at }}^{\prime}$ can be written in terms of the ground-state levels $\left|F, m_{F}\right\rangle$ only:

$\hat{H}_{\mathrm{at}}^{\prime}=\frac{\hat{\mathbf{p}}^{2}}{2 m}+V_{\mathrm{ext}}(\hat{\mathbf{x}})+\sum_{F, m_{F}} \hbar \omega_{F, m_{F}}\left|F, m_{F}\right\rangle\left\langle F, m_{F}\right|$,

where $\hat{\mathbf{p}}$ and $m$ are, respectively, the momentum and the mass of the atom, $V_{\text {ext }}(\hat{\mathbf{x}})$ is the trapping potential, which is kept fixed, and $\hbar \omega_{F, m_{F}}$ is the energy of the $\left|F, m_{F}\right\rangle$ atomic level, with $F$ denoting the hyperfine manifold and $m_{F}$ the Zeeman sublevel. In our experiment, the ${ }^{87} \mathrm{Rb}$ atoms are initialized in $\left|F=1, m_{F}=-1\right\rangle$, and near-resonant twophoton Raman transitions couple them to $\left|F=1, m_{F}=0\right\rangle$. Transitions to the $F=2$ manifold are off resonance by the hyperfine splitting $\omega_{\mathrm{HF}}=2 \pi \cdot 6.834 \mathrm{GHz}$ and can be neglected. The internal dynamics of each atom can then be described in terms of the spin operator $\hat{\mathbf{F}}=\left(\hat{F}_{x}, \hat{F}_{y}, \hat{F}_{z}\right)^{T}$, with $F=1$. The energy difference between the Zeeman sublevels is determined by first- and second-order Zeeman shifts $\hbar \omega_{z}^{(1)}<0$ and $\hbar \omega_{z}^{(2)}>0$, such that Eq. (B2) can be written as

$$
\hat{H}_{\mathrm{at}}^{\prime}=\frac{\hat{\mathbf{p}}^{2}}{2 m}+V_{\mathrm{ext}}(\hat{\mathbf{x}})+\hbar \omega_{z}^{(1)} \hat{F}_{z}+\hbar \omega_{z}^{(2)} \hat{F}_{z}^{2} .
$$

The Hamiltonian of the bare cavity mode reads

$$
\hat{H}_{\text {cav }}^{\prime}=\hbar \omega_{c} \hat{a}^{\dagger} \hat{a},
$$

where the operator $\hat{a}^{\dagger}$ creates $z$-polarized photons in the $\mathrm{TEM}_{00}$ cavity mode with resonance frequency $\omega_{c}$. In the dispersive regime at which we operate, the interaction between the light fields and the atom takes the form

$$
\hat{H}_{\mathrm{int}}^{\prime}=\alpha_{s} \hat{\mathbf{E}}^{(+)} \cdot \hat{\mathbf{E}}^{(-)}-i \frac{\alpha_{v}}{2 F}\left(\hat{\mathbf{E}}^{(+)} \times \hat{\mathbf{E}}^{(-)}\right) \cdot \hat{\mathbf{F}},
$$

where $\hat{\mathbf{E}}^{(-)}\left(\hat{\mathbf{E}}^{(+)}\right)$is the negative (positive) part of the total electric field at the position of the atom, with $\hat{\mathbf{E}}^{(+)}=\left(\hat{\mathbf{E}}^{(-)}\right)^{\dagger}$, and $\alpha_{s}$ and $\alpha_{v}$ are, respectively, the scalar and vectorial polarizability at the frequency of the driving lasers [48,78,79], with $\alpha_{s}<0$ and $\alpha_{v}>0$. In Eq. (B5), we are neglecting an additional rank-2 tensor contribution to the polarizability, which is justified for ${ }^{87} \mathrm{Rb}$ at the wavelength $\lambda=784.7 \mathrm{~nm}$ at which we operate.

We consider classical $y$-polarized transverse pump fields propagating along the $z$ direction at frequencies $\omega_{r, b}$ and a quantized cavity field. The negative part $\hat{\mathbf{E}}^{(-)}$of the total electric field is given by

$\hat{\mathbf{E}}^{(-)}=\frac{E_{r}}{2} f_{r}(\hat{\mathbf{x}}) \mathbf{e}_{y} e^{-i \omega_{r} t}+\frac{E_{b}}{2} f_{b}(\hat{\mathbf{x}}) \mathbf{e}_{y} e^{-i \omega_{b} t}+E_{0} g(\hat{\mathbf{x}}) \hat{a} \mathbf{e}_{z}$,

with unit vectors $\mathbf{e}_{j}(j \in\{x, y, z\})$ and spatial mode profiles $f_{r}(\hat{\mathbf{x}}), f_{b}(\hat{\mathbf{x}})$, and $g(\hat{\mathbf{x}})$. The two laser drives with amplitude $E_{r}$ and $E_{b}$ originate from the same optical fiber, and their standing-wave modulations overlap in phase at the position of the trapping potential. Given the small frequency difference $\omega_{b}-\omega_{r}=2 \pi \cdot 96 \mathrm{MHz}$, we can consider the same wave vector $k=\bar{\omega} / c$ for the two drives interacting with the atoms, with $\bar{\omega}=\left(\omega_{b}+\omega_{r}\right) / 2$, and restrict to a single spatial profile $f_{r}(\hat{\mathbf{x}})=f_{b}(\hat{\mathbf{x}})=f(\hat{\mathbf{x}})=$ $\exp \left[-2 x^{2} / w_{x}^{2}-2 y^{2} / w_{y}^{2}\right] \cos (k z)$. We also take $g(\hat{\mathbf{x}})=$ $\exp \left[-2\left(y^{2}+z^{2}\right) / w_{c}^{2}\right] \cos (k x)$ for the cavity mode profile.

We introduce the auxiliary Hamiltonian $\hat{H}_{\text {rot }}=$ $\hbar \bar{\omega} \hat{a}^{\dagger} \hat{a}-\hbar \omega_{z}^{\prime} \hat{F}_{z}$ and perform the unitary transformation $\hat{U}=\exp \left[(i / \hbar) \hat{H}_{\text {rot }} t\right]$, with $\omega_{z}^{\prime}=\left(\omega_{b}-\omega_{r}\right) / 2$. By making use of the rotating wave approximation, we obtain the timeindependent Hamiltonian 


$$
\hat{H}_{1}=\hat{H}_{\mathrm{at}}+\hat{H}_{\mathrm{cav}}+\hat{H}_{s}+\hat{H}_{v},
$$

where

$$
\hat{H}_{\mathrm{at}}=\frac{\hat{\mathbf{p}}^{2}}{2 m}+V_{\mathrm{ext}}(\hat{\mathbf{x}})+\hbar \delta_{z} \hat{F}_{z}+\hbar \omega_{z}^{(2)} \hat{F}_{z}^{2}
$$

and

$$
\hat{H}_{\mathrm{cav}}=-\hbar \Delta_{c} \hat{a}^{\dagger} \hat{a}
$$

with cavity detuning $\Delta_{c}=\bar{\omega}-\omega_{c}$ and effective linear shift $\delta_{z}=\omega_{z}^{(1)}+\omega_{z}^{\prime}$. The interaction part has a scalar and a vectorial contribution $\hat{H}_{s}$ and $\hat{H}_{v}$ given by

$$
\begin{aligned}
\hat{H}_{s}= & \frac{\alpha_{s}}{4}\left(E_{b}^{2}+E_{r}^{2}\right) f(\hat{\mathbf{x}})^{2}+\alpha_{s} E_{0}^{2} \hat{a}^{\dagger} \hat{a} g(\hat{\mathbf{x}})^{2}, \\
\hat{H}_{v}= & \frac{\alpha_{v}}{8} E_{0}\left[\left(E_{b}+E_{r}\right)\left(\hat{a}+\hat{a}^{\dagger}\right) \hat{F}_{x}\right. \\
& \left.+\left(E_{b}-E_{r}\right) i\left(\hat{a}-\hat{a}^{\dagger}\right) \hat{F}_{y}\right] f(\hat{\mathbf{x}}) g(\hat{\mathbf{x}}),
\end{aligned}
$$

respectively. Note that we apply a global rotation of the cavity field of the form $\hat{a} \rightarrow \hat{a} e^{i \pi / 2}$. The first term in the scalar interaction $\hat{H}_{s}$ [cf. Eq. (B10)] describes the attractive potential created by the transverse drives, giving rise to a one-dimensional lattice along the $z$ direction, with on-axis depth $V_{\mathrm{TP}}=-\alpha_{s}\left(E_{b}^{2}+E_{r}^{2}\right) / 4$, and to an additional confinement along the $x$ and $y$ direction. The second term in $\hat{H}_{s}$ is responsible for the dispersive shift of the cavity resonance and for a weak one-dimensional lattice potential along the $x$ direction. We define the maximal dispersive frequency shift per atom as $U_{0}=\alpha_{s} E_{0}^{2} / \hbar$. The vectorial interaction $\hat{H}_{v}$ in Eq. (B11) produces a spin-changing Raman transition between the Zeeman sublevels of the $F=1$ manifold. The spin-changing terms $\hat{F}_{x}$ and $\hat{F}_{y}$ are mediated by orthogonal quadratures of the cavity field and can be tuned by the sum or difference of the two pump fields, respectively.

\section{Many-body Hamiltonian}

We derive the Hamiltonian for the many-body system of $N$ atoms in a degenerate Bose gas using the secondquantization formalism. Using the single-atom results from the previous section, the many-body Hamiltonian can be written as

$\hat{H}_{\mathrm{MB}}=\hat{H}_{\mathrm{cav}}+\int \hat{\Psi}^{\dagger}(\mathbf{x})\left(\hat{H}_{\mathrm{at}}+\hat{H}_{s}+\hat{H}_{v}\right) \hat{\Psi}(\mathbf{x}) d \mathbf{x}$,

where the $\hat{\Psi}(\mathbf{x})$ is the spinor atomic field operator $\hat{\Psi}(\mathbf{x})=\left[\hat{\Psi}_{+1}(\mathbf{x}), \hat{\Psi}_{0}(\mathbf{x}), \hat{\Psi}_{-1}(\mathbf{x})\right]^{T}$, fulfilling the bosonic commutation relations $\left[\hat{\Psi}_{i}(\mathbf{x}), \hat{\Psi}_{j}^{\dagger}\left(\mathbf{x}^{\prime}\right)\right]=\delta_{i j} \delta\left(\mathbf{x}-\mathbf{x}^{\prime}\right)$ and
$\left[\hat{\Psi}_{i}(\mathbf{x}), \hat{\Psi}_{j}\left(\mathbf{x}^{\prime}\right)\right]=0$, with $i, j=+1,0,-1$. At this level, we neglect collisional interactions assuming low densities.

We set the half-frequency difference $\omega_{z}^{\prime}$ between the drives close to the energy separation between levels $\left|m_{F}=-1\right\rangle$ and $\left|m_{F}=0\right\rangle$, i.e., $\omega_{z}^{\prime} \approx \omega_{z}$, with $\omega_{z}=-\omega_{z}^{(1)}+\omega_{z}^{(2)}=$ $2 \pi \cdot 48 \mathrm{MHz}$. Thus, spin-changing Raman transitions to $\left|m_{F}=+1\right\rangle$ are off resonance by $\Delta_{+1} \approx 2 \omega_{z}^{(2)}=2 \pi \cdot 0.7 \mathrm{MHz}$. The large detuning $\Delta_{+1}$ determines the fastest timescale of the atomic evolution. This allows us to adiabatically eliminate the atomic operator $\hat{\Psi}_{+1}$ and restrict the dynamics to the two Zeeman sublevels with $m_{F}=0,-1$. The effect of the eliminated state leads to a subkilohertz energy shift of state $\left|m_{F}=0\right\rangle$, which we neglect.

We map our system to an effective generalized Dicke model by further restricting the Hilbert space to two spin-momentum modes, in the same spirit as previous works $[46,56]$. In the normal phase, the BEC, prepared in $m_{F}=-1$, occupies the ground state of the total trapping potential, resulting from the combination of the external trap $V_{\text {ext }}$ and the attractive lattice potential $V_{\mathrm{TP}}$ of the laser drives [cf. Eq (B10)]. We label this ground state as $\left|0_{a}\right\rangle$, with corresponding wave function $\Phi_{0_{a}}(\mathbf{x})$. The cavitymediated spin-changing interaction couples $\left|0_{a}\right\rangle$ to a density-modulated state $\left|1_{a}\right\rangle$ in $m_{F}=0$, with wave function $\Phi_{1_{a}}(\mathbf{x})=\mathcal{N} \Phi_{0_{a}}(\mathbf{x}) \cos (k x) \cos (k z)$, with $\mathcal{N}$ being a normalization factor. Within this two-mode description, the spinor field operator takes the form $\hat{\Psi}(\mathbf{x})=\left[0, \Phi_{1_{a}}(\mathbf{x}) \hat{c}_{1_{a}}\right.$, $\left.\Phi_{0_{a}}(\mathbf{x}) \hat{c}_{0_{a}}\right]^{T}$, where $\hat{c}_{0_{a}}$ and $\hat{c}_{1_{a}}$ are bosonic annihilation operators for the respective atomic modes. The corresponding expression for the many-body Hamiltonian is

$$
\begin{aligned}
\hat{H}_{\mathrm{MB}}= & -\hbar\left[\Delta_{c}-N \mathcal{I}\left(V_{\mathrm{TP}}\right) U_{0}\right] \hat{a}^{\dagger} \hat{a}+\hbar \omega_{0}\left(V_{\mathrm{TP}}\right) \hat{J}_{z} \\
& +\frac{\alpha_{v}}{4 \sqrt{2}} \mathcal{M}\left(V_{\mathrm{TP}}\right) E_{0}\left[\left(E_{b}+E_{r}\right)\left(\hat{a}+\hat{a}^{\dagger}\right) \hat{J}_{x}\right. \\
& \left.+i\left(E_{b}-E_{r}\right)\left(\hat{a}-\hat{a}^{\dagger}\right) \hat{J}_{y}\right],
\end{aligned}
$$

where we introduce collective pseudospin $N / 2$ operators $\hat{J}_{x}=\left(\hat{c}_{1_{a}}^{\dagger} \hat{c}_{0_{a}}+\hat{c}_{0_{a}}^{\dagger} \hat{c}_{1_{a}}\right) / 2, \hat{J}_{y}=\left(\hat{c}_{1_{a}}^{\dagger} \hat{c}_{0_{a}}-\hat{c}_{0_{a}}^{\dagger} \hat{c}_{1_{a}}\right) / 2 i$, and $\hat{J}_{z}=\left(\hat{c}_{1_{a}}^{\dagger} \hat{c}_{1_{a}}-\hat{c}_{0_{a}}^{\dagger} \hat{c}_{0_{a}}\right) / 2$. We indicate with $\hbar \omega_{0}\left(V_{\mathrm{TP}}\right)$ the energy difference between the bare atomic modes. The quantities $\mathcal{I}\left(V_{\mathrm{TP}}\right)$ and $\mathcal{M}\left(V_{\mathrm{TP}}\right)$ are overlap integrals defined by $\mathcal{I}\left(V_{\mathrm{TP}}\right)=\left\langle 0_{a}\left|g(\mathbf{x})^{2}\right| 0_{a}\right\rangle / N$ and $\mathcal{M}\left(V_{\mathrm{TP}}\right)=$ $\left\langle 0_{a}|f(\mathbf{x}) g(\mathbf{x})| 1_{a}\right\rangle / N$, respectively. In writing Eq. (B13), we neglect the dependence of the dispersive cavity shift on $\hat{J}_{z}$, which is a valid approximation whenever the system is not deep into the superradiant phase.

By considering $\hbar \omega_{0}\left(V_{\mathrm{TP}}\right)$ as a constant and taking its value in the limit of small $V_{\mathrm{TP}}$, i.e., $\hbar \omega_{0}=$ $\hbar\left(\omega_{z}^{\prime}-\omega_{z}+2 \omega_{\text {rec }}\right)$, we can write the Hamiltonian in Eq. (B13) in terms of the parameters defined in the main text: 


$$
\begin{aligned}
\hat{H}_{\mathrm{MB}}= & -\hbar \Delta_{c} \hat{a}^{\dagger} \hat{a}+\hbar \omega_{0} \hat{J}_{z}+\hbar\left(\eta_{b}+\eta_{r}\right)\left(\hat{a}+\hat{a}^{\dagger}\right) \hat{J}_{x} \\
& +i \hbar\left(\eta_{b}-\eta_{r}\right)\left(\hat{a}-\hat{a}^{\dagger}\right) \hat{J}_{y},
\end{aligned}
$$

where we use the substitution $\left[\Delta_{c}-N \mathcal{I}\left(V_{\mathrm{TP}}\right) U_{0}\right] \rightarrow \Delta_{c}$ and define $\hbar \eta_{b(r)}=\left(\alpha_{v} / 4 \sqrt{2}\right) \mathcal{M}\left(V_{\mathrm{TP}}\right) E_{0} E_{b(r)}$. Parametrizing Eq. (B14) in terms of $\bar{\eta}=\left(\eta_{b}+\eta_{r}\right) / 2$ and $\Delta \eta=$ $\left(\eta_{b}-\eta_{r}\right) / 2$ produces the Hamiltonian given in Eq. (1). A slight rearrangement of terms gives

$$
\begin{aligned}
\hat{H}_{\mathrm{MB}}= & -\hbar \Delta_{c} \hat{a}^{\dagger} \hat{a}+\hbar \omega_{0} \hat{J}_{z}+\hbar \eta_{b}\left(\hat{a} \hat{J}_{+}+\hat{a}^{\dagger} \hat{J}_{-}\right) \\
& +\hbar \eta_{r}\left(\hat{a}^{\dagger} \hat{J}_{+}+\hat{a} \hat{J}_{-}\right),
\end{aligned}
$$

with $\hat{J}_{ \pm}=\hat{J}_{x} \pm i \hat{J}_{y}$. From Eq. (B15), it is apparent that the couplings $\eta_{b}$ and $\eta_{r}$ tune the strength of the co- and counterrotating term of the light-matter interaction, respectively. In the limit $\eta_{r}=0$, the Hamiltonian (B15) reduces to the Tavis-Cummings model [80].

\section{Mapping between Hamiltonian couplings and experimental parameters}

We describe the mapping between the measured power of the transverse pump beams and the Raman couplings $\eta_{b}$ and $\eta_{r}$ introduced in the main text. The BEC is trapped in the combined potential of the harmonic confinement $V_{\text {ext }}(\mathbf{x})$ and of the attractive potential created by the transverse pumps $V_{\mathrm{TP}}(\mathbf{x})=-V_{\mathrm{TP}} f(\mathbf{x})^{2}$, which has contributions from the two drives, i.e., $V_{\mathrm{TP}}=V_{b}+V_{r}$. We monitor the power of each drive and extract the corresponding value of $V_{b(r)}$ in real time as described in the previous section.

To calculate the wave function $\Phi_{0_{a}}$, we consider spinindependent $s$-wave scattering and employ a ThomasFermi approximation for the interacting BEC in the total trapping potential [81]. Spin-changing collisions can be neglected due to the large second-order Zeeman shift $\omega_{z}^{(2)}=2 \pi \cdot 0.35 \mathrm{MHz}$ at which we operate [82]. In addition, we treat the one-dimensional lattice created by the transverse pump in the limit of large depth and approximate the lattice as a succession of independent harmonic traps. This is justified by the fact that, in our experiments, the superradiant phase transition occurs at large $V_{\text {TP }} \gtrsim 25 \hbar \omega_{\text {rec }}$. We then calculate the three-dimensional overlap integrals $\mathcal{I}\left(V_{\mathrm{TP}}\right)$ and $\mathcal{M}\left(V_{\mathrm{TP}}\right)$ defined in the previous subsection by using the expressions of the mode functions $f(\mathbf{x})=\exp \left[-2 x^{2} / w_{x}^{2}-2 y^{2} / w_{y}^{2}\right] \cos (k z)$ and $g(\mathbf{x})=\exp \left[-2\left(y^{2}+z^{2}\right) / w_{c}^{2}\right] \cos (k x)$, with waist sizes $\left[w_{x}, w_{y}, w_{c}\right]=[24,27,25] \mu \mathrm{m}$. For comparison, the estimated semiaxes of the modulated BEC near the superradiant transition are $[5.5,8.0,6.5] \mu \mathrm{m}$ in the three directions, respectively. The divergence of each mode over the extension of the BEC is negligible. The Raman couplings $\eta_{b}$ and $\eta_{r}$ are then found:

$$
\eta_{b(r)}=\frac{\mathcal{M}\left(V_{\mathrm{TP}}\right)}{2 \sqrt{2}} \frac{\alpha_{v}}{\operatorname{sgn}\left[\alpha_{s}\right] \cdot \alpha_{s}} \sqrt{-\frac{U_{0} V_{b(r)}}{\hbar}},
$$

where $\alpha_{v} / \alpha_{s}=-0.928$ and $\operatorname{sgn}\left[\alpha_{s}\right]=-1$ at the wavelength of the laser drives.

The overlap integral $\mathcal{M}\left(V_{\mathrm{TP}}\right)$ converges to $\mathcal{M}_{\max }=$ 0.68 at large lattice depths. In the regime $V_{\mathrm{TP}} \gtrsim 25 \hbar \omega_{\text {rec }}$ at which the superradiant phase transition occurs, $\mathcal{M}\left(V_{\mathrm{TP}}\right)$ deviates from $\mathcal{M}_{\max }$ by less than $2 \%$. For simplicity, we then assume $\mathcal{M}\left(V_{\mathrm{TP}}\right)=\mathcal{M}_{\max }$ when applying the conversion in Eq. (B16) throughout the paper.

\section{APPENDIX C: PHASE DIAGRAM}

For completeness, in this Appendix, we provide the mean-field steady-state solutions of the effective model (1). A similar treatment is conducted in Ref. [8]. The solutions are used to compute the analytical phase boundaries in Figs. 2 and 4.

\section{Analytical calculation of the steady state}

Starting from the Hamiltonian in Eq. (1), we consider dissipation due to photons leaking out from the cavity at rate $\kappa$ in the form of a Lindblad operator:

$$
\mathcal{L}[\hat{a}]=\kappa\left[2 \hat{a} \hat{\rho} \hat{a}^{\dagger}-\left\{\hat{a}^{\dagger} \hat{a}, \hat{\rho}\right\}\right] .
$$

We disregard the spin decay rate due to the negligible spontaneous emission between Zeeman sublevels. At this level, we also neglect the role of spin dephasing, which would lead to a negligible shift of the phase boundaries for our experimental parameters. Using the master equation

$$
\frac{d \hat{\rho}}{d t}=-\frac{i}{\hbar}[\hat{H}, \hat{\rho}]+\mathcal{L}[\hat{a}],
$$

we obtain mean-field equations of motion (EOMs) of the form

$$
\begin{aligned}
& \frac{d}{d t} \alpha=i \Delta_{c} \alpha-i 2 \sqrt{N} \bar{\eta} X-2 \sqrt{N} \Delta \eta Y-\kappa \alpha, \\
& \frac{d}{d t} X=-\omega_{0} Y-4 \sqrt{N} \Delta \eta \alpha_{\mathrm{Im}} Z, \\
& \frac{d}{d t} Y=\omega_{0} X-4 \sqrt{N} \bar{\eta} \alpha_{\mathrm{Re}} Z, \\
& \frac{d}{d t} Z=4 \sqrt{N} \bar{\eta} \alpha_{\mathrm{Re}} Y+4 \sqrt{N} \Delta \eta \alpha_{\mathrm{Im}} X,
\end{aligned}
$$

where the mean-field order parameters are $\langle\hat{a}\rangle=\sqrt{N} \alpha$, $\left\langle\hat{J}_{x}\right\rangle=N X,\left\langle\hat{J}_{y}\right\rangle=N Y$, and $\left\langle\hat{J}_{z}\right\rangle=N Z$. Introducing the renormalized couplings $\bar{\eta}_{N}=\sqrt{N} \bar{\eta}$ and $\Delta \eta_{N}=\sqrt{N} \Delta \eta$ and imposing the spin constraint $X^{2}+Y^{2}+Z^{2}=\frac{1}{4}$, we can solve analytically for $\alpha, X, Y$, and $Z$ in the steady state. The normal phase corresponds to the trivial steady-state 
solution $\alpha_{\operatorname{Re}}=\alpha_{\operatorname{Im}}=X=Y=0, \quad Z=-1 / 2$, with $\alpha_{\operatorname{Re}}=\operatorname{Re}[\alpha]$ and $\alpha_{\operatorname{Im}}=\operatorname{Im}[\alpha]$. The nontrivial solutions of Eq. (C3) [8] read

$$
\begin{aligned}
\alpha_{\mathrm{Re}} & = \pm \sqrt{c} \sqrt{\frac{2 a_{2}^{2} b_{1}+a_{2} b_{2}^{2}-2 a_{2} b_{3}\left(a_{1}+b_{1}\right)+2 a_{1} b_{3}^{2}+\operatorname{sgn}\left[\Delta \eta_{N}-\bar{\eta}_{N}\right] a_{2}\left|b_{2}\right| \sqrt{b_{2}^{2}-4\left(a_{1}-b_{1}\right)\left(a_{2}-b_{3}\right)}}{2\left[a_{2}^{2} b_{1}^{2}+a_{1}^{2} b_{3}^{2}+a_{1} a_{2}\left(b_{2}^{2}-2 b_{1} b_{3}\right)\right]},} \\
\alpha_{\mathrm{Im}} & =\frac{b_{2}^{2}-\operatorname{sgn}\left[\Delta \eta_{N}-\bar{\eta}_{N}\right]\left|b_{2}\right| \sqrt{4\left(b_{1}-a_{1}\right)\left(a_{2}-b_{3}\right)+b_{2}^{2}}}{2 b_{2}\left(a_{2}-b_{3}\right)} \alpha_{\operatorname{Re}}, \\
X & =-\frac{\Delta_{c} \alpha_{\mathrm{Re}}+\kappa \alpha_{\mathrm{Im}}}{2 \bar{\eta}_{N}}, \quad Y=\frac{\Delta_{c} \alpha_{\mathrm{Im}}-\kappa \alpha_{\mathrm{Re}}}{2 \Delta \eta_{N}}, \quad Z=\mathcal{A} \omega_{0},
\end{aligned}
$$

with

$$
\begin{aligned}
& a_{1}=16 \mathcal{A}^{2} \bar{\eta}_{N}^{2}, \quad a_{2}=16 \mathcal{A}^{2} \Delta \eta_{N}^{2}, \quad b_{1}=\left(\kappa^{2} / \Delta \eta_{N}^{2}+\Delta_{c}^{2} / \bar{\eta}_{N}^{2}\right) / 4, \\
& b_{2}=\kappa \Delta_{c}\left(1 / \bar{\eta}_{N}^{2}-1 / \Delta \eta_{N}^{2}\right) / 2, \quad b_{3}=\left(\kappa^{2} / \bar{\eta}_{N}^{2}+\Delta_{c}^{2} / \Delta \eta_{N}^{2}\right) / 4, \quad c=1 / 4-\mathcal{A}^{2} \omega_{0}^{2}, \\
& \mathcal{A}=-\frac{\left(\bar{\eta}_{N}^{2}+\Delta \eta_{N}^{2}\right) \Delta_{c}-\sqrt{\left(\bar{\eta}_{N}^{2}-\Delta \eta_{N}^{2}\right)^{2} \Delta_{c}^{2}-4 \kappa^{2} \bar{\eta}_{N}^{2} \Delta \eta_{N}^{2}}}{16 \bar{\eta}_{N}^{2} \Delta \eta_{N}^{2}} .
\end{aligned}
$$

\section{Analytic expressions for the phase boundaries}

We find an analytic expression for the slope of the dissipation-stabilized normal phase starting from the expression of $Z$ from Eq. (C4):

$Z=-\frac{\left(\bar{\eta}_{N}^{2}+\Delta \eta_{N}^{2}\right) \Delta_{c}-\sqrt{\left(\bar{\eta}_{N}^{2}-\Delta \eta_{N}^{2}\right)^{2} \Delta_{c}^{2}-4 \kappa^{2} \bar{\eta}_{N}^{2} \Delta \eta_{N}^{2}}}{16 \bar{\eta}_{N}^{2} \Delta \eta_{N}^{2}} \omega_{0}$

Requiring $Z$ to be real results in the condition

$$
\left(\bar{\eta}^{2}-\Delta \eta^{2}\right)^{2} \Delta_{c}^{2}-4 \kappa^{2} \bar{\eta}^{2} \Delta \eta^{2} \geq 0
$$

The equality provides the slope of the boundary between the superradiant phase and the dissipation-stabilized normal phase; cf. Fig. 2:

$$
(\Delta \eta / \bar{\eta})_{\mathrm{DSNP}}=\kappa / \Delta_{c}\left(1-\sqrt{1+\Delta_{c}^{2} / \kappa^{2}}\right)
$$

for $\Delta_{c}<0$. Moreover, from Eq. (C6) we also obtain the stability boundary of the normal phase. Specifically, in the normal phase, we set $Z=-1 / 2$ on the left-hand side of Eq. (C6) and square both sides; we then solve for $\Delta \eta_{N}$, obtaining the following expression:

$$
\Delta \eta_{N}=\frac{\sqrt{4 \bar{\eta}_{N}^{2}\left|\Delta_{c}\right| \omega_{0}-\omega_{0}^{2}\left(\Delta_{c}^{2}+\kappa^{2}\right)}}{2 \sqrt{4 \bar{\eta}_{N}^{2}-\omega_{0}\left|\Delta_{c}\right|}},
$$

which allows us to find the boundaries of the bistability region shown in Figs. 4(b) and 4(c) in the main text.

\section{Numerical simulations of the mean-field dynamics}

In order to simulate the time evolution of the system, we numerically solve the semiclassical EOMs in Appendix B, Eq. (B3). For this purpose, we use the MATLAB built-in "ode45" solver which is based on a Runge-Kutta $(4,5)$ method [83]. It employs variable time step sizes, and the error tolerance in each step is constrained to $10^{-8}$. To sample the fluctuations on top of the mean-field observables and allow for a phase transition to take place, we assume an initially small photon field of the form $\alpha(t=0)=[\operatorname{randn}(0,0.5)+i \cdot \operatorname{randn}(0,0.5)] / \sqrt{N} \quad$ with pseudorandom numbers randn $(0,0.5)$ sampled from a normal distribution with $(\mu, \sigma)=(0,0.5)$. This assumption is compatible with an initial coherent vacuum state for the cavity field, since $\left\langle\sqrt{N}\left(\alpha+\alpha^{*}\right) / 2\right\rangle_{S}=0$ and $\operatorname{var}_{S}\left[\sqrt{N}\left(\alpha+\alpha^{*}\right) / 2\right]=1 / 4$, where \langle\rangle$_{S}$ and $\operatorname{var}_{S}$ denote the average and variance, respectively, over a sufficiently large number of samples $S$.

\section{Measurement of the phase diagram}

\section{a. Experimental protocol and data processing}

To measure the phase diagram, we prepare a BEC in $m_{F}=-1$ and ramp up the power of the driving lasers at constant cavity detuning $\Delta_{c}$. The calibrated heterodyne signal is used to construct photon number spectrograms $\tilde{n}_{\mathrm{ph}}(f, t)$ as described in the next section. We integrate them in a narrow frequency range of $P=[\bar{\omega} / 2 \pi-$ $2.5 \mathrm{kHz}, \bar{\omega} / 2 \pi+2.5 \mathrm{kHz}]$ to obtain the photon traces $n_{\mathrm{ph}}(t)=\sum_{f \in P} \tilde{n}_{\mathrm{ph}}(f, t)$ of the cavity field at the frequency $\bar{\omega}$ characteristic of the superradiant phase.

The phase diagram in Fig. 2(b) of the main text is obtained by combining measurements for 51 different 

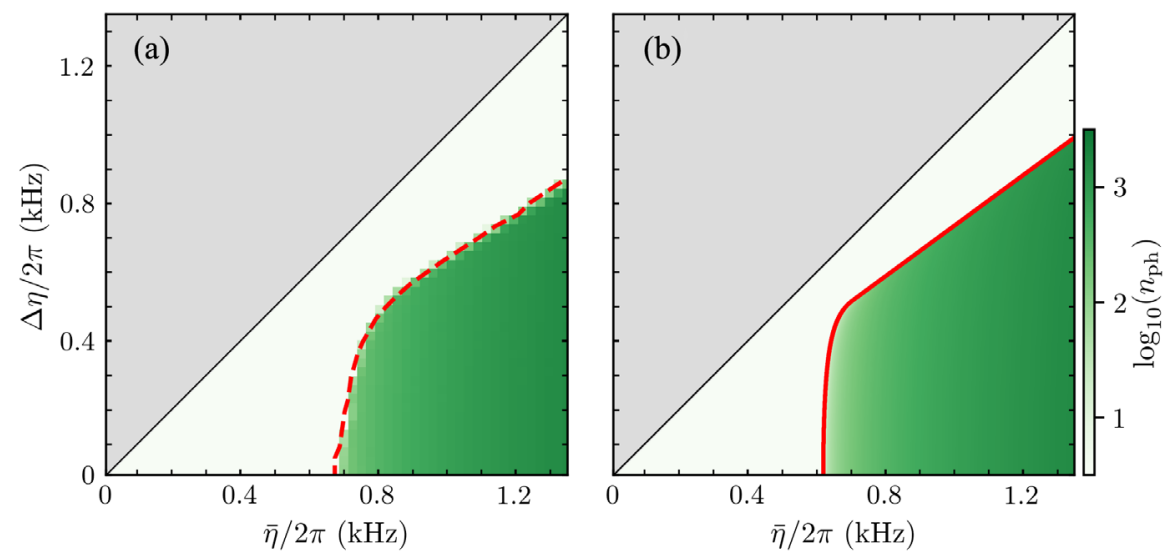

FIG. 5. Theoretical phase diagrams from numerical mean-field simulations (a) and analytic steady-state calculations (b). For both methods, we consider $\left(\omega_{0}, \Delta_{c}, \kappa\right)=2 \pi \cdot(44 \mathrm{kHz},-4.0 \mathrm{MHz}, 1.25 \mathrm{MHz})$ and $N=1.28 \times 10^{5}$ atoms, to match the experimental parameters. For the numerics, we carry out 427 simulations at 61 different coupling ratios $\Delta \eta / \bar{\eta}$ and use S-shaped coupling ramps with $t_{\text {ramp }}=10 \mathrm{~ms}$, as in the experiment. The boundary of the superradiant phase is marked in red.

ratios $\Delta \eta / \bar{\eta}$, with five realizations each. From each realization, we extract the coupling ramps $\bar{\eta}(t)$ and $\Delta \eta(t)$ by monitoring in real time the power of the driving lasers, as well as the time trace of the mean cavity photons $n_{\mathrm{ph}}(t)$ [cf. Fig. 2(a) in the main text]. After parametrizing $n_{\mathrm{ph}}$ as a function of $\bar{\eta}$ and $\Delta \eta$, the color plot in Fig. 2(b) of the main text is obtained by averaging the different experimental realizations in the $(\bar{\eta}, \Delta \eta)$ parameter space within squared bins with size $2 \pi \cdot 24 \mathrm{~Hz}$.

From each experimental realization, we extract the time $t_{\text {th }}$ at which the superradiant phase transition occurs by fitting $n_{\mathrm{ph}}(t)$ with a piecewise linear and power law function. The critical couplings are obtained as $\left(\bar{\eta}_{\text {th }}, \Delta \eta_{\text {th }}\right)=\left[\bar{\eta}\left(t_{\text {th }}\right), \Delta \eta\left(t_{\text {th }}\right)\right]$. The dots in Fig. 2(b) are the average of the critical couplings $\left(\bar{\eta}_{\mathrm{th}}, \Delta \eta_{\mathrm{th}}\right)$ from measurements taken with the same ratio $\Delta \eta / \bar{\eta}$. The error bars are the corresponding standard error of the mean.
The experimental boundary between superradiant phase and the dissipation-stabilized normal phase [dashed line in Fig. 2(b) in the main text] corresponds to the smallest value of $\Delta \eta / \bar{\eta}$ at which the phase transition is not observed in at least one of the experimental realizations, which we define as $(\Delta \eta / \bar{\eta})_{\text {DSNP. }}$. The upper (lower) boundary of the orange shaded region around this line marks the smallest (largest) ratio $\Delta \eta / \bar{\eta}$ at which the phase transition is absent (present) in all realizations. These bounds provide an uncertainty to the slope $(\Delta \eta / \bar{\eta})_{\text {DSNP. }}$.

\section{b. Theory comparison}

We plot in Fig. 5 the phase diagrams obtained from the numerical simulations and analytic steady-state calculations for the experimental parameters in Fig. 2 in the main text. The red lines in each plot indicate the boundaries of the superradiant phase assuming a threshold photon number of $n_{\mathrm{ph}, \mathrm{th}}=5$. We attribute the small shift of the
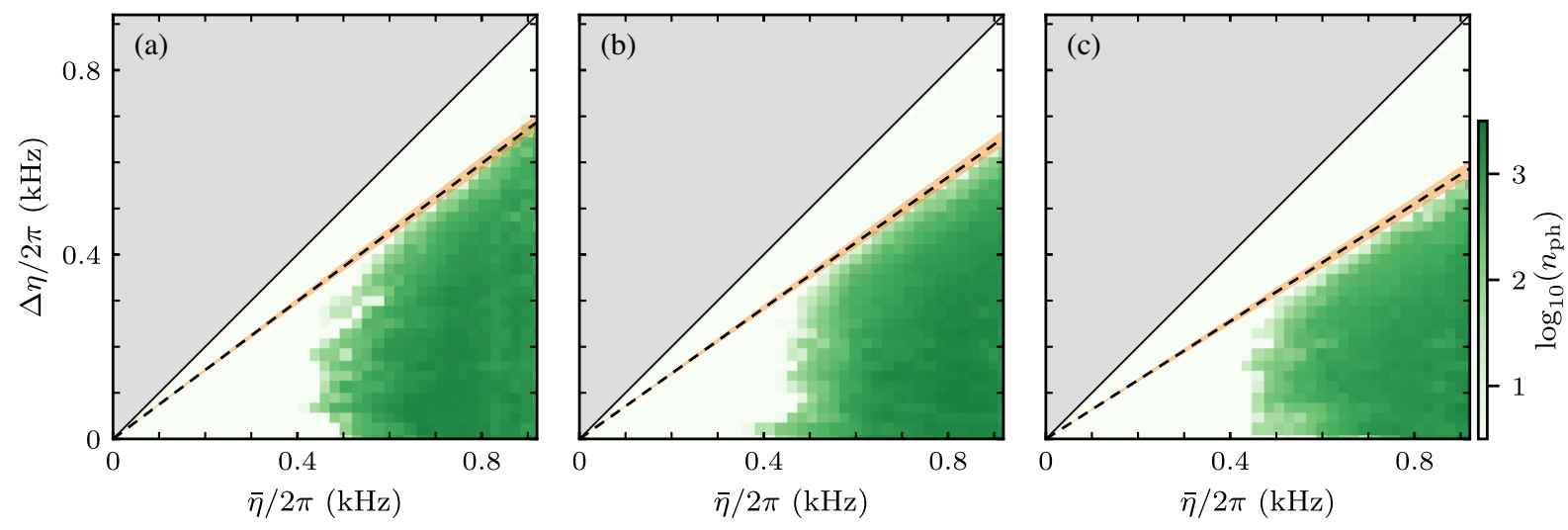

FIG. 6. Phase diagrams for different cavity detunings $\Delta_{c} / 2 \pi=-5.0(2)$ (a), $-4.0(2)$ (b), and $-3.0(2)$ MHz (c). For these measurements, $N=1.28(8) \times 10^{5}$ and $\omega_{0}=2 \pi \cdot 44(2) \mathrm{kHz}$. Each of the phase diagrams is a collection of 200-350 individual realizations with different coupling imbalances $\Delta \eta / \bar{\eta}$. The slope of the dashed line corresponds to $(\Delta \eta / \bar{\eta})_{\text {DSNP }}$ as described in the text. The orange shaded region around the dashed line marks the uncertainty on such a slope. 
phase boundaries of the numerical simulations from the analytical results to residual nonadiabatic effects.

\section{c. Phase diagrams for different cavity detunings}

We record experimental phase diagrams for three different cavity detunings $\Delta_{c}$ and display the results in Fig. 6. From each phase diagram, we extract the slope of the phase boundary $(\Delta \eta / \bar{\eta})_{\text {DSNP }}$ and its uncertainty as discussed above. The results of $(\Delta \eta / \bar{\eta})_{\text {DSNP }}$ vs $-\kappa / \Delta_{c}$ are shown in the inset in Fig. 2(b) in the main text.

\section{d. Absence of additional self-organization processes}

The drive at frequency $\omega_{r}$, red detuned from cavity resonance, may induce spurious atomic self-organization that does not involve a change of the $m_{F}$ state [56]. If present, this process is accompanied by the buildup of a coherent cavity field with the same polarization (along $y$ ) and frequency $\left(\omega_{r}\right)$ as the driving laser. During our measurements, we continuously monitor such a cavity field with the auxiliary heterodyne setup and never observe any signal above noise. The suppression of spin-preserving self-organization is due to the large detuning $\left|\omega_{r}-\omega_{c}\right|$ and to the presence of the second drive at frequency $\omega_{b}$.

\section{APPENDIX D: EXCITATION SPECTRUM}

In this Appendix, we detail the derivation of the excitation spectrum from our mean-field model. We then provide a theoretical background and numerical validation for the method used in Sec. IV to probe the properties of the polariton modes.

\section{Spectrum of the open system}

To find the spectrum of the open system, we consider the system's EOMs (C3) and expand the order parameters as $\alpha=\alpha_{0}+\delta \alpha, X=X_{0}+\delta X, Y=Y_{0}+\delta Y$, and $Z=Z_{0}+\delta Z$ around the steady states $\left(\alpha_{0}, X_{0}, Y_{0}, Z_{0}\right)$, chosen to be either the normal or the superradiant phase. A similar treatment is used in Refs. [7,8]. The linearized EOMs read

$$
\frac{d}{d t}\left(\begin{array}{c}
\delta \alpha_{\mathrm{Re}} \\
\delta \alpha_{\mathrm{Im}} \\
\delta X \\
\delta Y \\
\delta Z
\end{array}\right)=\mathbf{M}_{0}\left(\begin{array}{c}
\delta \alpha_{\mathrm{Re}} \\
\delta \alpha_{\mathrm{Im}} \\
\delta X \\
\delta Y \\
\delta Z
\end{array}\right),
$$

with

$$
\left.\begin{array}{ccc}
0 & -2 \Delta \eta_{N} & 0 \\
-2 \bar{\eta}_{N} & 0 & 0 \\
-\Gamma_{\phi} & -\omega_{0} & -4 \Delta \eta_{N} \alpha_{\mathrm{Im}}^{0} \\
\omega_{0} & -\Gamma_{\phi} & -4 \bar{\eta}_{N} \alpha_{\mathrm{Re}}^{0} \\
4 \Delta \eta_{N} \alpha_{\mathrm{Im}}^{0} & 4 \bar{\eta}_{N} \alpha_{\mathrm{Re}}^{0} & 0
\end{array}\right),
$$

where for completeness we phenomenologically include atomic dephasing at rate $\Gamma_{\phi}$. This damping term is compatible with a Lindblad term of the form $\mathcal{L}\left[\hat{J}_{z}\right]=$ $\Gamma_{\phi}\left[2 \hat{J}_{z} \hat{\rho} \hat{J}_{z}-\left\{\hat{J}_{z} \hat{J}_{z}, \hat{\rho}\right\}\right]$. From the diagonalization of the dynamical matrix in Eq. (D2), we obtain the eigenfrequencies and eigenmodes of the system around the steady states. Since in our experiment $\left|\Delta_{c}\right| \gg \omega_{0}$, a clear separation between the photonlike and atomlike polariton modes exists. The polariton mode $|1\rangle$ discussed in the main text is the atomlike mode obtained by linearizing around the normal phase. The eigenfrequencies associated to this polariton mode are the eigenvalues $\omega_{ \pm}$presented in Figs. 3(g) and 3(h). The $\omega_{-}$branch corresponds to the annihilation of a particle in the unexcited mode $|0\rangle$ and the creation of a particle in the polariton mode $|1\rangle$. The $\omega_{+}$ branch corresponds to the opposite process.

\section{Polaritonic decay rates $\gamma_{\downarrow(\uparrow)}$}

The decay rates $\gamma_{\downarrow(\uparrow)}$ of the low-energy polariton modes can be derived from the diagonalization of the dynamical matrix in Eq. (D2). Here, we derive the simplified analytical expression of $\gamma_{\downarrow(\uparrow)}$ given in Eq. (2) in the main text, which results from a perturbative expansion of the eigenvalues of the system in the small parameter $\omega_{0} / \kappa \ll 1$, which is well justified for our experiment. We do not consider spin dissipation and use the Keldysh action formulation [6,52]. First, we bosonize the spin using Holstein-Primakoff transformation, $\hat{S}^{z}=\hat{b}^{\dagger} \hat{b}-(N / 2)$, $\hat{S}^{+}=\sqrt{N-\hat{b}^{\dagger} \hat{b}} \hat{b}$, with $\hat{b}$ being a bosonic annihilation operator. We then write the Keldysh action in the frequency domain and integrate out the cavity degree of freedom, obtaining the spin-only action

$S_{\mathrm{spin}}=\int\left(\begin{array}{ll}\hat{\mathbf{b}}_{c}^{*} & \hat{\mathbf{b}}_{q}^{*}\end{array}\right)\left(\begin{array}{cc}0 & {\left[G_{A}^{\mathrm{spin}}\right]^{-1}} \\ {\left[G_{R}^{\mathrm{spin}}\right]^{-1}} & D_{K}^{\mathrm{spin}}\end{array}\right)\left(\begin{array}{l}\hat{\mathbf{b}}_{c} \\ \mathbf{b}_{q}\end{array}\right)$,

where the four-component Nambu spinor is given by $\hat{\mathbf{b}}_{i}=\left[\hat{b}_{i}(\omega) \hat{b}_{i}^{*}(-\omega)\right], i=c, q$, and the inverse Green's functions and Keldysh component are, respectively, 


$$
\begin{gathered}
{\left[G_{A}^{\mathrm{spin}}\right]^{-1}=\left(\left[G_{R}^{\mathrm{spin}}\right]^{-1}\right)^{\dagger}=\left(\begin{array}{cc}
\omega-\frac{N \eta_{b}^{2}}{i \kappa-\omega-\Delta_{c}}-\frac{N \eta_{r}^{2}}{-i \kappa+\omega-\Delta_{c}}-\omega_{0} & -\frac{2 N \eta_{b} \eta_{r} \Delta_{c}}{(\omega+i \kappa)^{2}-\Delta_{c}^{2}} \\
-\frac{2 N \eta_{b} \eta_{r} \Delta_{c}}{(\omega+i \kappa)^{2}-\Delta_{c}^{2}} & -\omega-\frac{N \eta_{b}^{2}}{-i \kappa+\omega-\Delta_{c}}-\frac{N \eta_{r}^{2}}{i \kappa-\omega-\Delta_{c}}-\omega_{0}
\end{array}\right),} \\
D_{K}^{\mathrm{spin}}=-2 i \kappa\left(\begin{array}{cc}
\frac{N \eta_{b}^{2}}{\kappa^{2}+\left(\omega+\Delta_{c}\right)^{2}}+\frac{N \eta_{b}^{2}}{\kappa^{2}+\left(\omega-\Delta_{c}\right)^{2}} & N \eta_{b} \eta_{r}\left(\frac{1}{\kappa^{2}+\left(\omega+\Delta_{c}\right)^{2}}+\frac{1}{\kappa^{2}+\left(\omega-\Delta_{c}\right)^{2}}\right) \\
N \eta_{b} \eta_{r}\left(\frac{1}{\kappa^{2}+\left(\omega+\Delta_{c}\right)^{2}}+\frac{1}{\kappa^{2}+\left(\omega-\Delta_{c}\right)^{2}}\right) & \frac{N \eta_{b}^{2}}{\kappa^{2}+\left(\omega-\Delta_{c}\right)^{2}}+\frac{N \eta_{b}^{2}}{\kappa^{2}+\left(\omega+\Delta_{c}\right)^{2}}
\end{array}\right) .
\end{gathered}
$$

We derive the eigenvalues as the zeros of the determinant of the inverse advanced Green's function in Eq. (D4):

$$
2 N \eta_{b}^{2}\left(-\Delta_{c} \omega_{0}-N \eta_{r}^{2}+i \kappa \omega+\omega^{2}\right)+\left[N \eta_{r}^{2}+\left(\omega_{0}-\omega\right)\left(-\Delta_{c}+i \kappa+\omega\right)\right]\left[N \eta_{r}^{2}+\left(\omega+\omega_{0}\right)\left(-\Delta_{c}-i \kappa-\omega\right)\right]+N^{2} \eta_{b}^{4}=0 .
$$

We perform a first-order expansion in $\omega_{0} / \kappa \ll 1$ by approximating $\omega+i \kappa \approx i \kappa$ and solve Eq. (D6) for $\omega$. We obtain

$$
\begin{aligned}
\omega_{ \pm} & \approx i N \frac{\kappa}{\kappa^{2}+\Delta_{c}^{2}}\left(\eta_{b}^{2}-\eta_{r}^{2}\right) \pm \frac{\sqrt{\left[-4 N \Delta \eta^{2} \Delta_{c}+\left(\kappa^{2}+\Delta_{c}^{2}\right) \omega_{0}\right]\left[-4 N \bar{\eta}^{2} \Delta_{c}+\left(\kappa^{2}+\Delta_{c}^{2}\right) \omega_{0}\right]}}{\kappa^{2}+\Delta_{c}^{2}} \\
& =-i\left(\gamma_{\downarrow}-\gamma_{\uparrow}\right) \pm \omega_{0} \sqrt{\left(1-\frac{\bar{\eta}^{2}}{\eta_{c}^{2}}\right)\left(1-\frac{\Delta \eta^{2}}{\eta_{c}^{2}}\right)} .
\end{aligned}
$$

We note that this result can be obtained also from a linear analysis after adiabatic elimination of the cavity field. The rates $\gamma_{\downarrow(\uparrow)}$ describe the dissipative damping (amplification) of the polariton mode $|1\rangle$, as discussed in the main text. They can be rewritten in the form

$$
\gamma_{\downarrow(\uparrow)}=N \eta_{b(r)}^{2} \rho(\bar{\omega}),
$$

where $\rho(\bar{\omega})=\kappa /\left[\left(\bar{\omega}-\omega_{c}\right)^{2}+\kappa^{2}\right]$ is the density of states of the cavity at the frequency $\bar{\omega}$ of the cavity field; cf. Fig. 1. The expression (D9) indicates that the mechanism at the origin of damping (amplification) $\gamma_{\downarrow(\uparrow)}$ is the scattering of photons from a single drive with strength $\eta_{b(r)}$ into the bath of vacuum modes provided by the cavity, accompanied by a transfer of population from mode $|1\rangle(|0\rangle)$ to mode $|0\rangle(|1\rangle)$.

The expression in Eq. (D9) is valid in the limit $\omega_{0} \ll \kappa$. A more accurate estimation for $\gamma_{\downarrow(\uparrow)}$ can be obtained using Fermi's golden rule [51] and the limit in which only a single drive is present, i.e., $\eta_{b}=0$ or $\eta_{r}=0$. The result is

$$
\gamma_{\downarrow(\uparrow)}=N \eta_{b(r)}^{2} \rho\left(\tilde{\omega}_{+(-)}\right),
$$

with $\tilde{\omega}_{ \pm}=\bar{\omega} \pm \omega_{0}$. The frequency of the field scattered into the cavity by each drive deviates from $\bar{\omega}$ by $\pm \omega_{0}$, according to energy conservation [see also Fig. 1(c) for a schematic visualization].

The correction obtained by using Fermi's golden rule becomes particularly relevant near the Dicke limit $\eta_{b}=\eta_{r}$. Without this correction, the rates $\gamma_{\downarrow(\uparrow)}$ compensate each other [cf. Eq. (D9)], and the imaginary part of Eq. (D8) is zero for all $\bar{\eta}<\bar{\eta}_{c}$, resulting in a vanishing damping rate. The result obtained with Fermi's golden rule allows us to account for higher orders of $\omega_{0} / \kappa$, leading to a nonzero damping. By plugging Eq. (D10) into the expression for the eigenvalues Eq. (D8), we find that, in the Dicke limit, the effective damping rate of the polariton mode $|1\rangle$ is

$$
\gamma_{\downarrow}-\gamma_{\uparrow}=-4 N \eta_{b}^{2} \frac{\kappa \Delta_{c} \omega_{0}}{\left(\Delta_{c}^{2}+\kappa^{2}\right)^{2}},
$$

where we again make use of $\omega_{0} \ll \kappa$. This result is in agreement with previous derivations [84] and provides insights into the physical origin of a finite effective polariton damping in the driven-dissipative Dicke model.

\section{Nonlocal vs local dissipation}

Dissipation is present in our system as photon losses affecting the cavity mode, which is modeled by the Lindblad superoperator $\hat{\mathcal{L}}[\hat{\rho}]=\kappa\left[2 \hat{a} \hat{\rho} \hat{a}^{\dagger}-\left\{\hat{a}^{\dagger} \hat{a}, \hat{\rho}\right\}\right]$. From the point of view of the atomic ensemble, this constitutes a nonlocal dissipation channel [85], as the individual pseudospins are collectively coupled to the bath via the coupling to the same cavity mode $\hat{a}$. The nonlocal character of dissipation is crucial to obtain the results presented in this work. We elucidate this point by comparing our system to an analogous model featuring bidirectional local dissipation. For simplicity, in this subsection, we neglect the motional degrees of freedom of the atoms. For the auxiliary 
model with local dissipation, we consider the same Hamiltonian as in Eq. (1) and a Lindblad superoperator $\hat{\mathcal{L}}_{\Gamma}[\hat{\rho}]=\Gamma_{\uparrow} \sum_{i=1}^{N} \mathcal{D}\left[\hat{s}_{i}^{+}\right]+\Gamma_{\downarrow} \sum_{i=1}^{N} \mathcal{D}\left[\hat{s}_{i}^{-}\right], \quad$ with $\quad \mathcal{D}[\hat{x}]=$ $2 \hat{x} \hat{\rho} \hat{x}^{\dagger}-\left\{\hat{x}^{\dagger} \hat{x}, \hat{\rho}\right\}$ and where $\hat{s}_{i}^{-}$and $\hat{s}_{i}^{+}$are the jump operators for the pseudospin of a single atom $i$. Such a form of dissipation could be obtained, for instance, by enhancing the spontaneous emission from the optically excited levels in our Raman scheme; cf. Fig. 1(c) [86]. This gives rise to dissipative transfers of population from state $\left|0_{a}\right\rangle\left(\left|1_{a}\right\rangle\right)$ to $\left|1_{a}\right\rangle\left(\left|0_{a}\right\rangle\right)$ controlled by the rate $\Gamma_{\uparrow}\left(\Gamma_{\downarrow}\right)$.

Although the gain-loss competition in this scenario looks similar to the one of our system, in which the dissipative rates $\gamma_{\uparrow}$ and $\gamma_{\downarrow}$ appear, the overall effect of dissipation is substantially different. As described in the previous paragraph, in the Dicke limit $\Delta \eta=0$ the decay rates $\gamma_{\uparrow}$ and $\gamma_{\downarrow}$ compensate each other (to first order), giving rise to an overall polariton damping $\operatorname{Im}\left[\omega_{ \pm}\right] \approx \gamma_{\uparrow}-\gamma_{\downarrow}=0$ below the critical point $\bar{\eta}<\eta_{c}$ [cf. Eq. (2)]. This compensation is at the heart of our capability to tune the effective dissipation, and eventually to minimize it, by acting on the relative strength of the two Raman drives. In contrast, spontaneous emission leads to an overall polariton damping $\operatorname{Im}\left[\omega_{ \pm}\right] \approx$ $\Gamma_{\uparrow}+\Gamma_{\downarrow}$ for $\bar{\eta}<\eta_{c}$ [86]. The two counteracting processes $\Gamma_{\downarrow}$ and $\Gamma_{\uparrow}$ cannot compensate each other.

The different behavior of the two models reflects the local or nonlocal character of the coupling to the bath. A quantum jump due to spontaneous emission projects the atomic ensemble to a state with reduced total pseudospin $|\hat{\vec{J}}|<N / 2$, i.e., leads to a reduced coherence within the system. Conversely, a quantum jump due to photon loss from the cavity mode does not lead to any local projection and preserves the spin coherence, i.e., $|\hat{\vec{J}}|=N / 2$.

\section{Probing excitations}

\section{a. Experimental protocol}

To measure the evolution of the excitation spectra, we prepare a BEC of $N=9.6(4) \times 10^{4}$ atoms in $m_{F}=-1$ and ramp up the coupling strengths $\eta_{r, b}(t)$ within $t_{\text {ramp }}=$ $9.1 \mathrm{~ms}$. The experimental parameters for these measurements are $\omega_{0}=2 \pi \cdot 48(4) \mathrm{kHz}$ and $\Delta_{c}=-2 \pi \cdot 5.8(1) \mathrm{MHz}$. While ramping up the coupling, we inject an excitation field through the cavity between $t \in[3.0,4.0] \mathrm{ms}$. The excitation field is derived from the same laser source at $784.7 \mathrm{~nm}$ as the transverse drives and the local oscillator for the heterodyne setup. Its frequency can be independently tuned via a double-pass AOM. The amplitude of the excitation field corresponds to 7.2(1) intracavity photons; its frequency is chosen to be close to the polariton resonance $\bar{\omega}+\omega_{0}$. By this method, we typically transfer $<10 \%$ of the atomic population in the excited polariton mode $|1\rangle$. After the end of the excitation pulse, the polariton mode evolves freely according to the dynamics of the open system. We monitor this free evolution in real time via the spectrum of the associated photon field. The relative phase between the excitation field and the Raman drives is not actively stabilized and is subject to slow drifts between different experimental realizations, due to changes in the optical paths. This relative phase influences the transfer efficiency to the excited mode $|1\rangle$, similarly to Bragg spectroscopy, but does not affect the free evolution of the excitation in time and frequency.

\section{b. Relation between the polariton dynamics and the cavity spectrogram}

Here, we show how the dynamics of the polaritonic excitation can be derived from the associated cavity field, detected with a heterodyne setup, as done in Sec. IV. Since the population in mode $|1\rangle$ prepared by the excitation pulse is small, we can linearize the mean-field dynamics of the system around the normal phase. By substituting $\left(\alpha_{\mathrm{Re}}, \alpha_{\mathrm{Im}}, X_{0}, Y_{0}, Z_{0}\right)=(0,0,0,0,-1 / 2) \quad$ in Eq. (D2) and linearizing the pseudospin conservation $\delta Z=$ $-\left(X_{0} \delta X+Y_{0} \delta Y\right) / Z_{0}=0$, we obtain a $4 \times 4$ stability matrix $\mathbf{M}$ in the basis $\left(\delta \alpha_{\mathrm{Re}}, \delta \alpha_{\mathrm{Im}}, \delta X, \delta Y\right)^{T}$. Diagonalization of this matrix leads to

$$
\mathbf{D}=\mathbf{S}^{-1} \mathbf{M S}=\left(\begin{array}{cccc}
-i \Delta_{+} & 0 & 0 & 0 \\
0 & -i \Delta_{-} & 0 & 0 \\
0 & 0 & -i \omega_{+} & 0 \\
0 & 0 & 0 & -i \omega_{-}
\end{array}\right)
$$

where $\Delta_{+}=-\Delta_{-}^{*}$ and $\omega_{+}=-\omega_{-}^{*}$ because $\mathbf{M}$ has real coefficients. The pairs $\Delta_{ \pm}, \omega_{ \pm}$correspond to the photonlike and atomlike polariton modes, respectively, with $\left|\operatorname{Re}\left[\Delta_{ \pm}\right]\right| \approx$ $\left|\Delta_{c}\right|$ and $\left|\operatorname{Re}\left[\omega_{ \pm}\right]\right| \leq \omega_{0}$ [87]. The eigenfrequencies depend on the couplings $\bar{\eta}$ and $\Delta \eta$, which are time dependent in our experimental protocol. The coupling sweeps are, however, slow enough to allow the system to evolve adiabatically, and the polariton modes evolve independently of each other. By decomposing into polariton modes, the evolution of the cavity field quadratures can be written as

$$
\alpha_{\operatorname{Re}}(t)=\delta \alpha_{\operatorname{Re}}(t)=\sum_{j= \pm} c_{p j} e^{i \Delta_{j} t}+\sum_{j= \pm} c_{a j} e^{i \omega_{j} t}
$$

where $c_{l j}$ are complex coefficients, with $c_{l+}=c_{l-}^{*}$ and $l=p, a$ denoting photon and atom, respectively. Because of the large separation $\left|\Delta_{c}\right| \gg \omega_{0}$, the photonlike mode cannot be excited by the external pulse on resonance with the atomlike mode and can be neglected. We, thus, find

$$
\alpha_{\operatorname{Re}}(t)=c_{0} \cos \left(\operatorname{Re}\left[\omega_{+}\right] t+\phi_{0}\right) e^{\operatorname{Im}\left[\omega_{+}\right] t} .
$$

The initial conditions $c_{0}$ and $\phi_{0}$ are determined by the externally induced excitation process. An analogous expression holds for $\alpha_{\mathrm{Im}}$, allowing one to directly relate the real and imaginary part of the polariton frequency to 
the measured cavity output. Specifically, from Eq. (D14), we find that, since $\operatorname{Im}\left[\omega_{ \pm}\right]<0$, the amplitude of the field $n_{\mathrm{ph}}$ decays as $n_{\mathrm{ph}} \propto e^{2 \operatorname{Im}\left[\omega_{ \pm}\right] t}$, with a corresponding $1 / e$-decay time $\tau=-\left(2 \operatorname{Im}\left[\omega_{ \pm}\right] t\right)^{-1}$, which is the result used in Sec. IV.

\section{c. Data processing and comparison to theory}

The experimental values of the excitations lifetime shown in Fig. 3(f) of the main text are extracted from the heterodyne measurement of the cavity output in the following way. We consider the photon number spectrogram $\tilde{n}_{\mathrm{ph}}(\omega, t)$ and set $t=0$ to the end of the excitation pulse. We first extract the time $t_{c}$ at which the superradiant phase transition occurs by integrating $\tilde{n}_{\mathrm{ph}}(\omega, t)$ on a frequency interval $-\omega_{\lim } \leq \omega-\bar{\omega} \leq \omega_{\lim }$ with $\omega_{\lim }=2 \pi$. $10 \mathrm{kHz} \approx 0.2 \omega_{0}$ and setting a transition threshold of 100 intracavity photons. Such a threshold is large enough to capture only the coherent field in the superradiant phase but still small enough to detect reliably the critical point. For large ratios $\Delta \eta / \bar{\eta}$ leading to the dissipation-stabilized normal phase, $t_{c}$ is taken as the average of the transition times extracted where the superradiant phase builds up. To study the time evolution of the polaritonic excitations, we integrate $\tilde{n}_{\mathrm{ph}}(\omega, t)$ on a larger frequency range $\omega_{\text {lim }}=2 \pi$. $150 \mathrm{kHz} \sim 3 \omega_{0}$ to get the photon trace $n_{\mathrm{ph}}(t)$, with a time resolution of $10 \mu \mathrm{s}$. We extract the lifetime $\tau$ from the cumulative signal $s(t)=\int_{0}^{t} n_{\mathrm{ph}}\left(t^{\prime}\right) d t^{\prime}$. For $n_{\mathrm{ph}} \propto e^{-t / \tau}, s(t)$ takes the form $s(t)=s_{\max }\left(1-e^{-t / \tau}\right)$. Since for most of the data points the lifetime is significantly shorter than the transition time $t_{c}$, we determine $\tau$ by the time at which $s(t)$ reaches a fraction $\left(1-e^{-1}\right)$ of its maximum below $t_{c}$. The results of this estimation are in agreement with the ones obtained from a fit of $s(t)$ but more robust especially for weak signals. As an exception, for the single dataset at $\Delta \eta / \bar{\eta}=0$ the condition $\tau \ll t_{c}$ is not fulfilled, and $\tau$ is extracted from a fit of $s(t)$ with the exponential model. We neglect the experimental realizations in which the atomic response during the excitation pulse is below the noise level. The data shown in Fig. 3(f) of the main text are averaged values of $\tau$ over 10-25 realizations, with the error bar representing the maximum between standard error of the mean and the time resolution of the photon trace.

We compare the values of $\tau$ extracted experimentally with the theoretical expectations from the excitation eigenfrequencies of the system. According to the description given in Appendix $\mathrm{C}$, we expect that $n_{\mathrm{ph}}(t) \propto e^{2 \operatorname{Im}\left[\omega_{ \pm}\right] t}$, which provides an analytical estimation of the lifetime $\tau_{\text {an }}=-\left(2 \operatorname{Im}\left[\omega_{ \pm}\right]\right)^{-1}$. If the couplings vary in time, the decay of the photon number $n_{\mathrm{ph}}(t)$ is, in general, nonexponential. However, a meaningful estimation for the measured lifetime $\tau$ at large enough imbalance ratio $\Delta \eta / \bar{\eta} \gtrsim 0.05$ is provided by the value $\tau_{\text {an }}$ obtained for the instantaneous couplings $\bar{\eta}$ and $\Delta \eta$ just after the excitation pulse [88].

To account for processes leading to dephasing of the individual atomic spins, such as collisions, we introduce a phenomenological atomic dephasing rate $\Gamma_{\phi}$, as described in Appendix C. In Fig. 3(f) of the main text, the blue shaded region shows the lifetime estimated from the eigenvalues and assuming $\Gamma_{\phi}=0$ (upper bound) and $\Gamma_{\phi}=2 \pi \cdot 500 \mathrm{~Hz}$ (lower bound, corresponding to the estimated collision rate in the total trapping potential).

For a closer comparison to the experiment, a numerical simulation including time-varying coupling is performed, using the method described in Appendix $\mathrm{C}$ [red shaded region in Fig. 3(f) of the main text]. The shaded region includes results of simulations performed for different initial phase $\phi_{\text {probe }} \in[0,2 \pi)$ of the excitation drive, which we do not control in the experiment, and an atomic dephasing rate $\Gamma_{\phi}$ varying in the same interval described in the previous paragraph.

\section{Numerical simulations of the probing method}

We simulate numerically the experimental protocol that we implement to probe the excitation spectrum of our system and described in Sec. IV. For this purpose, we extend the theoretical model and incorporate an additional intracavity probe beam. We consider a classical $z$-polarized electric field propagating along the cavity axis:

$$
E_{\text {probe }}(t, x)=\tilde{E}_{\text {probe }} \sqrt{n_{\text {probe }}(t)} \cos \left(k_{\text {rec }} x\right) e^{-i \omega_{\text {probe }} t-i \phi_{\text {probe }}},
$$

with $\omega_{\text {probe }}=\bar{\omega}+\omega_{0}+\delta_{\text {probe }}$. Here, $n_{\text {probe }}(t), \phi_{\text {probe }}$, and $\delta_{\text {probe }}$ are, respectively, the average intracavity photon number, relative phase, and detuning with respect to the cavity field associated with the polariton branch $\omega_{+}$at low couplings $\left(\bar{\omega}+\omega_{0}\right)$. Moreover, $\tilde{E}_{\text {probe }}$ is the electric field per photon in this beam.

Following an analogous approach to the derivation of the Hamiltonian $\hat{H}$ in Appendix B, we obtain a time-dependent many-body Hamiltonian describing the interaction of the light-matter system with the probe field:

$$
\begin{aligned}
\hat{H}_{\text {exc }}= & \hat{H}_{\mathrm{MB}}+4 \hbar \bar{\eta} \sqrt{n_{\text {probe }}(t)} \sin \left[\left(\omega_{0}+\delta_{\text {probe }}\right) t+\phi_{\text {probe }}\right] \hat{J}_{x} \\
& +4 \hbar \Delta \eta \sqrt{n_{\text {probe }}(t)} \cos \left[\left(\omega_{0}+\delta_{\text {probe }}\right) t+\phi_{\text {probe }}\right] \hat{J}_{y} .
\end{aligned}
$$

The probe beam drives the atomic coherences $\hat{J}_{x, y}$, similar to cavity-enhanced Bragg spectroscopy techniques [89]. Hence, we expect to coherently transfer non-negligible atomic populations to the excited state if we approach the low-coupling two-photon resonance $\delta_{\text {probe }} \rightarrow 0$. 


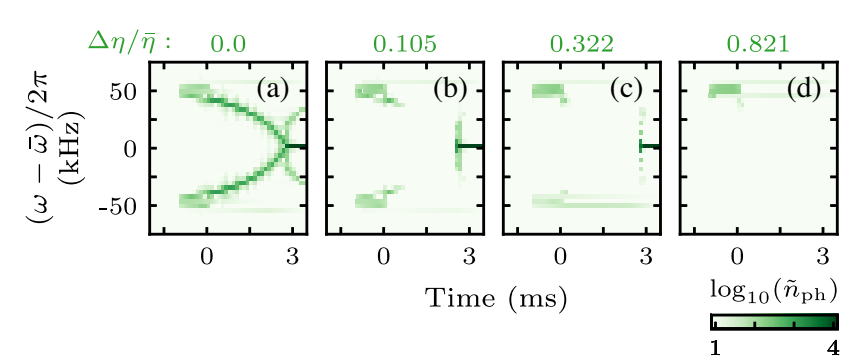

FIG. 7. Spectrograms of the cavity field from numerical simulations of our experimental protocol to probe excitations; cf. Fig. 3. We consider the coupling imbalances $\Delta \eta / \bar{\eta}=0$ (a), 0.105 (b), 0.322 (c), and 0.821 (d). For the numerical simulation, we choose $\left(\omega_{0}, \omega, \kappa, \Gamma\right)=2 \pi \cdot(48 \mathrm{kHz}, 5.8 \mathrm{MHz}, 1.25 \mathrm{MHz}$, $100 \mathrm{~Hz}$ ) and $N=9.6 \times 10^{4}$ atoms. The couplings are increased via an $\mathrm{S}$-shaped ramp within $9.1 \mathrm{~ms}$ to $\bar{\eta}<2 \pi \cdot 1.16 \mathrm{kHz}$ at fixed ratio $\Delta \eta / \bar{\eta}$. Moreover, a blue-detuned probe with $\delta_{\text {probe }}=2 \pi \cdot 2 \mathrm{kHz}, n_{\text {probe }}=7.2$ photons, and $\phi_{\text {probe }}=0$ illuminates the system between $-1 \mathrm{~ms}<t<0 \mathrm{~ms}$.

We derive mean-field equations of motion from the Hamiltonian in Eq. (D16) and numerically solve them to simulate the experimental conditions of our excitation probing method; cf. Fig. 3. We plot the resulting spectrograms of the PSD in Fig. 7. The coupling imbalances $\Delta \eta / \bar{\eta}$ and the parameters $\left(\omega_{0}, \Delta_{c}, \kappa, N\right)$ are chosen in accordance with the experimental observations in Figs. 3(b)-3(e). Depending on the choice of $\Delta \eta / \bar{\eta}$, we transfer between $8 \%$ and $12 \%$ of the atoms to the excited state at the end of the probe pulse. The results from the numerical simulations are in good agreement with the experimental results at different coupling imbalances: While in the Dicke limit the excitations are long lived and a complete mode softening toward the superradiant phase is observed, the polaritonic excitations damp faster for increasingly large coupling imbalances, as observed in the experiment.

\section{APPENDIX E: HYSTERESIS MEASUREMENT}

\section{Data processing}

For every single hysteresis measurement, we fix the coupling strength $\bar{\eta}$ and record the photon number $n_{\mathrm{ph}}$ extracted from the heterodyne detector as a function of the other coupling strength $\Delta \eta$. To further process the raw data, we smoothen it by applying a moving average over 51 subsequent points. Subsequently, we define a threshold photon number for the detection of a stable superradiant phase.

In order to extract the threshold of the superradiant region, we set a threshold of 36 mean photons, which is 5 times above the noise level. Around this value, the width of the hysteresis is independent on the choice of the threshold. We determine and compare the critical coupling strength for the forward (backward) path, as shown in purple (orange), in Fig. 4 of the main text and in Fig. 8 below. The critical couplings are used to map out the

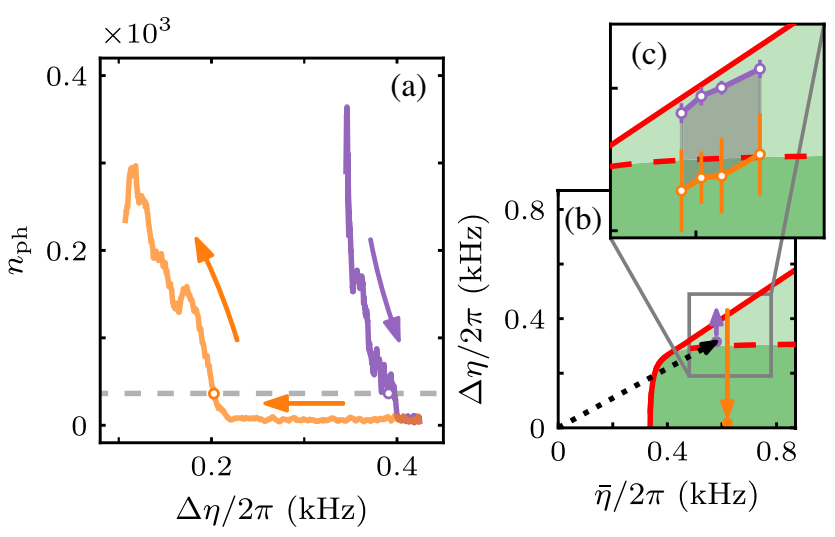

FIG. 8. Hysteresis at the boundary between the superradiant phase and the dissipation-stabilized normal phase. The hysteresis is measured starting in the superradiant phase and performing a loop of the coupling $\Delta \eta$ in the opposite direction as the one shown in the main text. (a) Representative time trace of the mean photon number $n_{\mathrm{ph}}$ during the loop. (b) Corresponding trajectory in the parameter space $(\bar{\eta}, \Delta \eta)$. An artificial offset in $\bar{\eta}$ is introduced between the forward and backward paths for better visibility. (c) Boundaries of the normal phase detected during the forward (purple) and backward (orange) path for different $\bar{\eta}$. The position of the boundaries is determined from the photon traces by setting a threshold of 36 mean intracavity photons, as indicated with a gray line in (a). The data points are mean values of 12-20 realizations, with the error bars representing the standard deviation. As a guide to the reader, in the background in (b),(c), the phase diagram from analytical calculations shows the region of stable normal phase (white), stable superradiant phase (dark green), and bistability (light green). The theoretical boundaries are rescaled to the experimental data, with a single factor applied to both couplings. This scaling factor is chosen to overlay the theoretical phase boundary between the superradiant and bistability regions (dashed red line) and the corresponding experimental data point with the largest coupling $\bar{\eta}$. For these measurements, we employ the following experimental parameters: $N=1.10(8) \times 10^{5}, \Delta_{c}=-2 \pi \cdot 3.0(5) \mathrm{MHz}$, and $\omega_{0}=2 \pi \cdot 40(5) \mathrm{kHz}$.

hysteresis region for different coupling strengths $\bar{\eta}$. For every data point, we take on average 15 measurements, with at least 12 and at most 18 repetitions.

\section{Hysteresis loops in opposite directions}

Our hysteresis measurement is potentially sensitive to atom loss and heating during the experimental protocol. These processes affect the collective atom-cavity coupling and, consequently, shift the stability boundaries of the different phases. To ensure that the measured bistability region is not substantially biased by a variation of the collective coupling due to these effects, we complement the measurement shown in Fig. 4 of the main text with the result of a hysteresis loop performed in the opposite direction; see Fig. 8. Hysteresis is observed also in this second measurement protocol, confirming that the effect of atom loss and heating is not substantial. 
[1] S. Sachdev, Quantum Phase Transitions, 2nd ed. (Cambridge University Press, Cambridge, England, 2011).

[2] L. D. Carr, Understanding Quantum Phase Transitions (Taylor \& Francis, London, 2010).

[3] M. Müller, S. Diehl, G. Pupillo, and P. Zoller, in Advances in Atomic, Molecular, and Optical Physics, Vol. 61, edited by P. Berman, E. Arimondo, and C. Lin (Academic, New York, 2012), pp. 1-80.

[4] A. J. Daley, Quantum Trajectories and Open Many-Body Quantum Systems, Adv. Phys. 63, 77 (2014).

[5] I. Carusotto and C. Ciuti, Quantum Fluids of Light, Rev. Mod. Phys. 85, 299 (2013).

[6] L. M. Sieberer, M. Buchhold, and S. Diehl, Keldysh Field Theory for Driven Open Quantum Systems, Rep. Prog. Phys. 79, 096001 (2016).

[7] M. Soriente, T. L. Heugel, K. Arimitsu, R. Chitra, and O. Zilberberg, Distinctive Class of Dissipation-Induced Phase Transitions and Their Universal Characteristics, Phys. Rev. Research 3, 023100 (2021).

[8] M. Soriente, T. Donner, R. Chitra, and O. Zilberberg, Dissipation-Induced Anomalous Multicritical Phenomena, Phys. Rev. Lett. 120, 183603 (2018).

[9] S. Diehl, E. Rico, M. A. Baranov, and P. Zoller, Topology by Dissipation in Atomic Quantum Wires, Nat. Phys. 7, 971 (2011).

[10] J. T. Barreiro, M. Müller, P. Schindler, D. Nigg, T. Monz, M. Chwalla, M. Hennrich, C. F. Roos, P. Zoller, and R. Blatt, An Open-System Quantum Simulator with Trapped Ions, Nature (London) 470, 486 (2011).

[11] Y. Lin, J. P. Gaebler, F. Reiter, T. R. Tan, R. Bowler, A. S. Sørensen, D. Leibfried, and D. J. Wineland, Dissipative Production of a Maximally Entangled Steady State of Two Quantum Bits, Nature (London) 504, 415 (2013).

[12] H. Krauter, C. A. Muschik, K. Jensen, W. Wasilewski, J. M. Petersen, J. I. Cirac, and E. S. Polzik, Entanglement Generated by Dissipation and Steady State Entanglement of Two Macroscopic Objects, Phys. Rev. Lett. 107, 080503 (2011).

[13] I. Bloch, J. Dalibard, and W. Zwerger, Many-Body Physics with Ultracold Gases, Rev. Mod. Phys. 80, 885 (2008).

[14] M. Lewenstein, A. Sanpera, and V. Ahufinger, Ultracold Atoms in Optical Lattices (Oxford University, New York, 2012), p. 479.

[15] T. Langen, R. Geiger, and J. Schmiedmayer, Ultracold Atoms out of Equilibrium, Annu. Rev. Condens. Matter Phys. 6, 201 (2015).

[16] R. Labouvie, B. Santra, S. Heun, and H. Ott, Bistability in a Driven-Dissipative Superfluid, Phys. Rev. Lett. 116, 235302 (2016).

[17] F. Letscher, O. Thomas, T. Niederprüm, M. Fleischhauer, and H. Ott, Bistability versus Metastability in Driven Dissipative Rydberg Gases, Phys. Rev. X 7, 021020 (2017).

[18] M. Fitzpatrick, N. M. Sundaresan, A. C. Y. Li, J. Koch, and A. A. Houck, Observation of a Dissipative Phase Transition in a One-Dimensional Circuit QED Lattice, Phys. Rev. X 7, 011016 (2017).

[19] T. Fink, A. Schade, S. Höfling, C. Schneider, and A. Imamoglu, Signatures of a Dissipative Phase Transition in Photon Correlation Measurements, Nat. Phys. 14, 365 (2018).
[20] T. Tomita, S. Nakajima, I. Danshita, Y. Takasu, and Y. Takahashi, Observation of the Mott Insulator to Superfluid Crossover of a Driven-Dissipative Bose-Hubbard System, Sci. Adv. 3, e1701513 (2017).

[21] R. Ma, B. Saxberg, C. Owens, N. Leung, Y. Lu, J. Simon, and D. I. Schuster, A Dissipatively Stabilized Mott Insulator of Photons, Nature (London) 566, 51 (2019).

[22] O. Morsch and I. Lesanovsky, Dissipative Many-Body Physics of Cold Rydberg Atoms, Riv. Nuovo Cimento Soc. Ital. Fis. 41, 383 (2018).

[23] N. Dogra, M. Landini, K. Kroeger, L. Hruby, T. Donner, and T. Esslinger, Dissipation-Induced Structural Instability and Chiral Dynamics in a Quantum Gas, Science 366, 1496 (2019).

[24] H. Keßler, P. Kongkhambut, C. Georges, L. Mathey, J. G. Cosme, and A. Hemmerich, Observation of a Dissipative Time Crystal, Phys. Rev. Lett. 127, 043602 (2021).

[25] P. Bienias, J. Douglas, A. Paris-Mandoki, P. Titum, I. Mirgorodskiy, C. Tresp, E. Zeuthen, M. J. Gullans, M. Manzoni, S. Hofferberth, D. Chang, and A. V. Gorshkov, Photon Propagation through Dissipative Rydberg Media at Large Input Rates, Phys. Rev. Research 2, 033049 (2020).

[26] D. P. Ornelas-Huerta, P. Bienias, A. N. Craddock, M. J. Gullans, A. J. Hachtel, M. Kalinowski, M. E. Lyon, A. V. Gorshkov, S. L. Rolston, and J. V. Porto, Tunable ThreeBody Loss in a Nonlinear Rydberg Medium, Phys. Rev. Lett. 126, 173401 (2021).

[27] F. E. Öztürk, T. Lappe, G. Hellmann, J. Schmitt, J. Klaers, F. Vewinger, J. Kroha, and M. Weitz, Observation of a NonHermitian Phase Transition in an Optical Quantum Gas, Science 372, 88 (2021).

[28] D. N. Basov, R. D. Averitt, and D. Hsieh, Towards Properties on Demand in Quantum Materials, Nat. Mater. 16, 1077 (2017).

[29] T. Byrnes, N. Y. Kim, and Y. Yamamoto, Exciton-Polariton Condensates, Nat. Phys. 10, 803 (2014).

[30] A. Cavalleri, Photo-induced Superconductivity, Contemp. Phys. 59, 31 (2018).

[31] S. Smolka, W. Wuester, F. Haupt, S. Faelt, W. Wegscheider, and A. Imamoglu, Cavity Quantum Electrodynamics with Many-Body States of a Two-Dimensional Electron Gas, Science 346, 332 (2014).

[32] E. Orgiu, J. George, J. A. Hutchison, E. Devaux, J. F. Dayen, B. Doudin, F. Stellacci, C. Genet, J. Schachenmayer, C. Genes, G. Pupillo, P. Samorì, and T. W. Ebbesen, Conductivity in Organic Semiconductors Hybridized with the Vacuum Field, Nat. Mater. 14, 1123 (2015).

[33] G. Mazza and A. Georges, Superradiant Quantum Materials, Phys. Rev. Lett. 122, 017401 (2019).

[34] Y. Ashida, A. Imamoglu, J. Faist, D. Jaksch, A. Cavalleri, and E. Demler, Quantum Electrodynamic Control of Matter: Cavity-Enhanced Ferroelectric Phase Transition, Phys. Rev. X 10, 041027 (2020).

[35] J. B. Curtis, Z. M. Raines, A. A. Allocca, M. Hafezi, and V. M. Galitski, Cavity Quantum Eliashberg Enhancement of Superconductivity, Phys. Rev. Lett. 122, 167002 (2019).

[36] H. Gao, F. Schlawin, M. Buzzi, A. Cavalleri, and D. Jaksch, Photoinduced Electron Pairing in a Driven Cavity, Phys. Rev. Lett. 125, 053602 (2020). 
[37] I. Carusotto, A. A. Houck, A. J. Kollár, P. Roushan, D. I. Schuster, and J. Simon, Photonic Materials in Circuit Quantum Electrodynamics, Nat. Phys. 16, 268 (2020).

[38] H. Ritsch, P. Domokos, F. Brennecke, and T. Esslinger, Cold Atoms in Cavity-Generated Dynamical Optical Potentials, Rev. Mod. Phys. 85, 553 (2013).

[39] F. Mivehvar, F. Piazza, T. Donner, and H. Ritsch, Cavity QED with Quantum Gases: New Paradigms in Many-Body Physics, arXiv:2102.04473.

[40] K. Hammerer, A. S. Sørensen, and E. S. Polzik, Quantum Interface between Light and Atomic Ensembles, Rev. Mod. Phys. 82, 1041 (2010).

[41] J. Simon, H. Tanji, S. Ghosh, and V. Vuletić, Single-Photon Bus Connecting Spin-Wave Quantum Memories, Nat. Phys. 3, 765 (2007).

[42] Z. Zhiqiang, C. H. Lee, R. Kumar, K. J. Arnold, S. J. Masson, A. S. Parkins, and M. D. Barrett, Nonequilibrium Phase Transition in a Spin-1 Dicke Model, Optica 4, 424 (2017).

[43] E. J. Davis, G. Bentsen, L. Homeier, T. Li, and M. H. Schleier-Smith, Photon-Mediated Spin-Exchange Dynamics of Spin-1 Atoms, Phys. Rev. Lett. 122, 010405 (2019).

[44] M. A. Norcia, R. J. Lewis-Swan, J. R. K. Cline, B. Zhu, A. M. Rey, and J. K. Thompson, Cavity-Mediated Collective Spin-Exchange Interactions in a Strontium Superradiant Laser, Science 361, 259 (2018).

[45] J. A. Muniz, D. Barberena, R. J. Lewis-Swan, D. J. Young, J. R. K. Cline, A. M. Rey, and J. K. Thompson, Exploring Dynamical Phase Transitions with Cold Atoms in an Optical Cavity, Nature (London) 580, 602 (2020).

[46] R. M. Kroeze, Y. Guo, V. D. Vaidya, J. Keeling, and B. L. Lev, Spinor Self-Ordering of a Quantum Gas in a Cavity, Phys. Rev. Lett. 121, 163601 (2018).

[47] J. Kohler, J. A. Gerber, E. Dowd, and D. M. Stamper-Kurn, Negative-Mass Instability of the Spin and Motion of an Atomic Gas Driven by Optical Cavity Backaction, Phys. Rev. Lett. 120, 013601 (2018).

[48] M. Landini, N. Dogra, K. Kroeger, L. Hruby, T. Donner, and T. Esslinger, Formation of a Spin Texture in a Quantum Gas Coupled to a Cavity, Phys. Rev. Lett. 120, 223602 (2018).

[49] P. Kirton, M. M. Roses, J. Keeling, and E. G. Dalla Torre, Introduction to the Dicke Model: From Equilibrium to Nonequilibrium, and Vice Versa, Adv. Quantum Technol. 2, 1800043 (2019).

[50] K. C. Stitely, A. Giraldo, B. Krauskopf, and S. Parkins, Nonlinear Semiclassical Dynamics of the Unbalanced, Open Dicke Model, Phys. Rev. Research 2, 033131 (2020).

[51] Y. Shchadilova, M. M. Roses, E. G. Dalla Torre, M. D. Lukin, and E. Demler, Fermionic Formalism for DrivenDissipative Multilevel Systems, Phys. Rev. A 101, 013817 (2020).

[52] M. Soriente, R. Chitra, and O. Zilberberg, Distinguishing Phases Using the Dynamical Response of DrivenDissipative Light-Matter Systems, Phys. Rev. A 101, 023823 (2020).

[53] Z. Zhang, C. H. Lee, R. Kumar, K. J. Arnold, S. J. Masson, A. L. Grimsmo, A. S. Parkins, and M. D. Barrett, DickeModel Simulation via Cavity-Assisted Raman Transitions, Phys. Rev. A 97, 043858 (2018).
[54] R. H. Dicke, Coherence in Spontaneous Radiation Processes, Phys. Rev. 93, 99 (1954).

[55] F. Dimer, B. Estienne, A. S. Parkins, and H. J. Carmichael, Proposed Realization of the Dicke-Model Quantum Phase Transition in an Optical Cavity QED System, Phys. Rev. A 75, 013804 (2007).

[56] K. Baumann, C. Guerlin, F. Brennecke, and T. Esslinger, Dicke Quantum Phase Transition with a Superfluid Gas in an Optical Cavity, Nature (London) 464, 1301 (2010).

[57] G. Vrijsen, O. Hosten, J. Lee, S. Bernon, and M. A. Kasevich, Raman Lasing with a Cold Atom Gain Medium in a High-Finesse Optical Cavity, Phys. Rev. Lett. 107, 063904 (2011).

[58] J. G. Bohnet, Z. Chen, J. M. Weiner, D. Meiser, M. J. Holland, and J. K. Thompson, A Steady-State Superradiant Laser with Less than One Intracavity Photon, Nature (London) 484, 78 (2012).

[59] J. G. Bohnet, Z. Chen, J. M. Weiner, K. C. Cox, and J. K. Thompson, Linear-Response Theory for Superradiant Lasers, Phys. Rev. A 89, 013806 (2014).

[60] Y. Zhang, Y.-X. Zhang, and K. Mølmer, Monte-Carlo Simulations of Superradiant Lasing, New J. Phys. 20, 112001 (2018).

[61] K. Baumann, R. Mottl, F. Brennecke, and T. Esslinger, Exploring Symmetry Breaking at the Dicke Quantum Phase Transition, Phys. Rev. Lett. 107, 140402 (2011).

[62] F. Brennecke, R. Mottl, K. Baumann, R. Landig, T. Donner, and T. Esslinger, Real-Time Observation of Fluctuations at the Driven-Dissipative Dicke Phase Transition, Proc. Natl. Acad. Sci. U.S.A. 110, 11763 (2013).

[63] D. Nagy, G. Szirmai, and P. Domokos, Self-Organization of a Bose-Einstein Condensate in an Optical Cavity, Eur. Phys. J. D 48, 127 (2008).

[64] H. Eleuch and I. Rotter, Width Bifurcation and Dynamical Phase Transitions in Open Quantum Systems, Phys. Rev. E 87, 052136 (2013).

[65] H. Lipkin, N. Meshkov, and A. Glick, Validity of ManyBody Approximation Methods for a Solvable Model: (i). Exact Solutions and Perturbation Theory, Nucl. Phys. 62, 188 (1965).

[66] S. Morrison and A. S. Parkins, Dynamical Quantum Phase Transitions in the Dissipative Lipkin-Meshkov-Glick Model with Proposed Realization in Optical Cavity QED, Phys. Rev. Lett. 100, 040403 (2008).

[67] F. Damanet, A. J. Daley, and J. Keeling, Atom-Only Descriptions of the Driven-Dissipative Dicke Model, Phys. Rev. A 99, 033845 (2019).

[68] J. S. Ferreira and P. Ribeiro, Lipkin-Meshkov-Glick Model with Markovian Dissipation: A Description of a Collective Spin on a Metallic Surface, Phys. Rev. B 100, 184422 (2019).

[69] P. Titum and M. F. Maghrebi, Nonequilibrium Criticality in Quench Dynamics of Long-Range Spin Models, Phys. Rev. Lett. 125, 040602 (2020).

[70] T. E. Lee, F. Reiter, and N. Moiseyev, Entanglement and Spin Squeezing in Non-Hermitian Phase Transitions, Phys. Rev. Lett. 113, 250401 (2014).

[71] J. S. Huber, G. Rastelli, M. J. Seitner, J. Kölbl, W. Belzig, M. I. Dykman, and E. M. Weig, Spectral Evidence of 
Squeezing of a Weakly Damped Driven Nanomechanical Mode, Phys. Rev. X 10, 021066 (2020).

[72] R. Ramírez, M. Reboiro, and D. Tielas, Exceptional Points from the Hamiltonian of a Hybrid Physical System: Squeezing and Anti-squeezing, Eur. Phys. J. D 74, 193 (2020).

[73] K. C. Stitely, S. J. Masson, A. Giraldo, B. Krauskopf, and S. Parkins, Superradiant Switching, Quantum Hysteresis, and Oscillations in a Generalized Dicke Model, Phys. Rev. A 102, 063702 (2020).

[74] T. L. Heugel, M. Biondi, O. Zilberberg, and R. Chitra, Quantum Transducer Using a Parametric DrivenDissipative Phase Transition, Phys. Rev. Lett. 123, 173601 (2019).

[75] Y. Deng, J. Cheng, H. Jing, and S. Yi, Bose-Einstein Condensates with Cavity-Mediated Spin-Orbit Coupling, Phys. Rev. Lett. 112, 143007 (2014).

[76] R. M. Kroeze, Y. Guo, and B. L. Lev, Dynamical Spin-Orbit Coupling of a Quantum Gas, Phys. Rev. Lett. 123, 160404 (2019).

[77] B. Gadway, D. Pertot, R. Reimann, M. G. Cohen, and D. Schneble, Analysis of Kapitza-Dirac Diffraction Patterns beyond the Raman-Nath Regime, Opt. Express 17, 19173 (2009).

[78] N. Goldman, G. Juzeliūnas, P. Öhberg, and I. B. Spielman, Light-Induced Gauge Fields for Ultracold Atoms, Rep. Prog. Phys. 77, 126401 (2014).

[79] F. Le Kien, P. Schneeweiss, and A. Rauschenbeutel, Dynamical Polarizability of Atoms in Arbitrary Light Fields: General Theory and Application to Cesium, Eur. Phys. J. D 67, 92 (2013).

[80] M. Tavis and F. W. Cummings, Exact Solution for an n-Molecule-Radiation-Field Hamiltonian, Phys. Rev. 170, 379 (1968).
[81] F. Dalfovo, S. Giorgini, L. P. Pitaevskii, and S. Stringari, Theory of Bose-Einstein Condensation in Trapped Gases, Rev. Mod. Phys. 71, 463 (1999).

[82] D. M. Stamper-Kurn and M. Ueda, Spinor Bose Gases: Symmetries, Magnetism, and Quantum Dynamics, Rev. Mod. Phys. 85, 1191 (2013).

[83] L. F. Shampine and M.W. Reichelt, The MATLAB ODE suite, SIAM J. Sci. Comput. 18, 1 (1997).

[84] M. J. Bhaseen, J. Mayoh, B. D. Simons, and J. Keeling, Dynamics of Nonequilibrium Dicke Models, Phys. Rev. A 85, 013817 (2012).

[85] K. Seetharam, A. Lerose, R. Fazio, and J. Marino, Correlation Engineering via Non-local Dissipation, arXiv:2101 .06445 .

[86] P. Kirton and J. Keeling, Superradiant and Lasing States in Driven-Dissipative Dicke Models, New J. Phys. 20, 015009 (2018).

[87] D. Nagy, G. Szirmai, and P. Domokos, Critical Exponent of a Quantum-Noise-Driven Phase Transition: The OpenSystem Dicke Model, Phys. Rev. A 84, 043637 (2011).

[88] Using the knowledge of the coupling ramps $\bar{\eta}(t)$ and $\Delta \eta(t)$, we parametrize $\tau_{\text {an }}$ as a function of time $\tau_{\text {an }}=\tau_{\text {an }}(t)$. We compare the lifetime after the excitation pulse $\tau_{\text {an }}(t=0)$ with its variation $\delta \tau$ within the decay time of the excitations, estimated as $\delta \tau=\left[\tau_{\text {an }}(0)-\tau_{\text {an }}\left(t_{b}\right) / t_{b}\right] \tau_{\text {an }}$, where $t_{b}$ is the time corresponding to the bifurcation. For $\Gamma_{\phi}=0$ and large enough imbalance ratios $\Delta \eta / \bar{\eta}>0.05$, we get $\delta \tau / \tau_{\text {an }}<0.1$. Closer to the Dicke limit, the estimation is sensitive to the assumption on the atomic dephasing rate $\Gamma_{\phi}$, and the condition $\delta \tau / \tau_{\text {an }} \ll 1$ is fulfilled only for large values of $\Gamma_{\phi}$.

[89] R. Mottl, F. Brennecke, K. Baumann, R. Landig, T. Donner, and T. Esslinger, Roton-Type Mode Softening in a Quantum Gas with Cavity-Mediated Long-Range Interactions, Science 336, 1570 (2012). 